\title{
DETERMINATION OF THIABENDAZOLE IN ORANGE JUICE USING AN MISPE-SERS \\ CHEMOSENSOR \\ by
}

Jingyi Feng

B.Sc. with honors, St. Francis Xavier University, 2014

A THESIS SUBMITTED IN PARTIAL FULFILLMENT OF THE REQUIREMENTS FOR THE DEGREE OF

\author{
MASTER OF SCIENCE \\ in \\ THE FACULTY OF GRADUATE AND POSTDOCTORAL STUDIES \\ (Chemistry)
}

THE UNIVERSITY OF BRITISH COLUMBIA

(Vancouver)

February 2017

C Jingyi Feng, 2016 


\begin{abstract}
Thiabendazole is a systemic fungicide frequently used to treat vegetables and fruits during the postharvest process, leading to potential detriment to consumers. This work has combined molecularly imprinted polymers (MIPs) with surface enhanced Raman spectroscopy (SERS) to afford a novel MISPE-SERS chemosensor to determine trace amount of thiabendazole in orange juice. MIPs were synthesized via precipitation polymerization. Kinetic and static adsorption tests validated the efficient separation and enrichment of thiabendazole by using MIPs. Synthesized MIPs were packed into solid phase extraction (SPE) cartridge to work as tailor-made sorbents for the separation of thiabendazole in orange juice. Silver colloid functioned as SERS-active substrate to quantify eluted thiabendazole by MISPE-SERS chemosensor. The whole process only took 23 min with limit of detection of 4 ppm for thiabendazole. In sum, this MISPE-SERS chemosensor can be considered as a promising method for fast and high-throughput detection of trace amount of thiabendazole in agri-food products.
\end{abstract}




\section{Preface}

The work presented in this thesis is original, independent work by myself.

Chapter 3 "Determination of thiabendazole in orange juice using an MISPE-SERS chemosensor" will be submitted as a full paper prepared with Prof. Xiaonan Lu and Prof. Ed Grant. 


\section{Table of Contents}

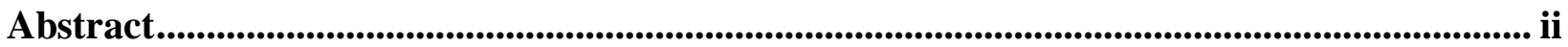

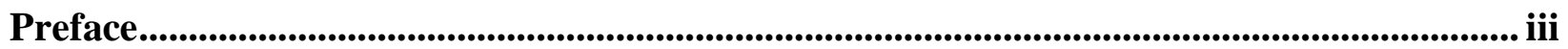

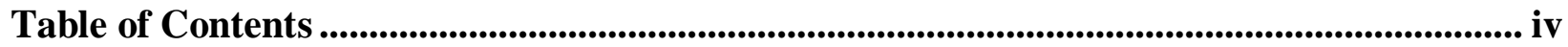

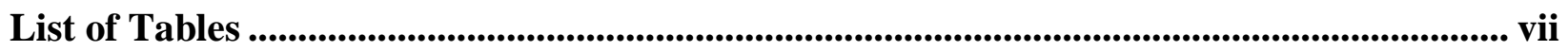

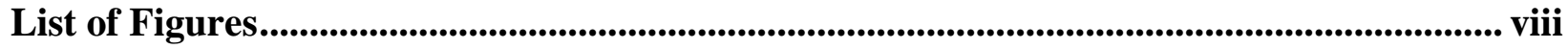

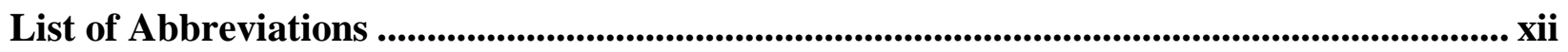

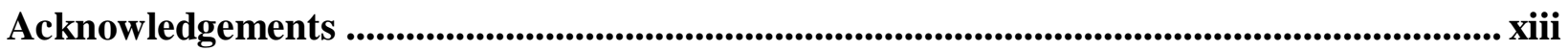

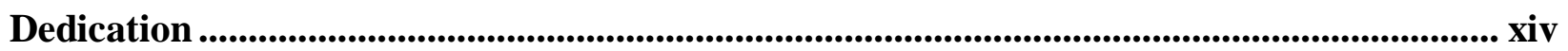

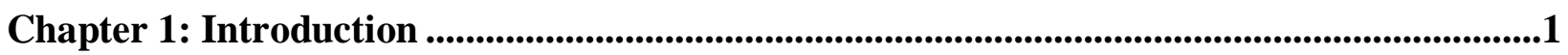

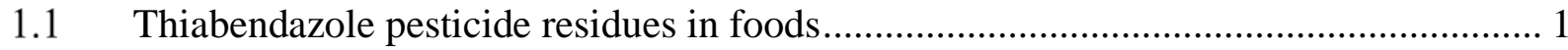

1.2 History of molecularly imprinting technique.................................................. 2

1.2.1 General mechanism of molecular imprinting ............................................. 3

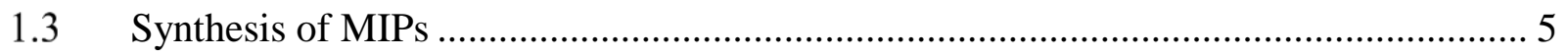

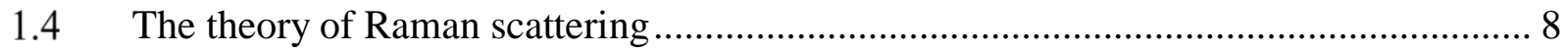

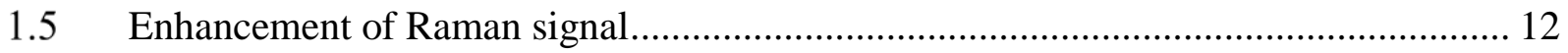

1.5.1 Resonance Raman (RR) spectroscopy …................................................... 12

1.5.2 Surface-enhanced Raman spectroscopy (SERS) ......................................... 13

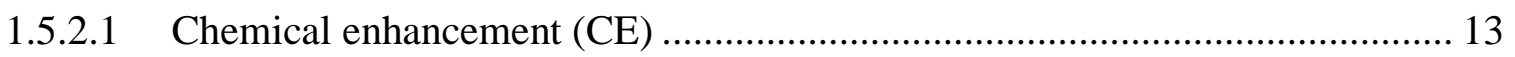

1.5.2.2 Electromagnetic field enhancement (EM) ........................................... 14

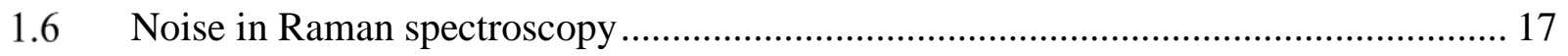

1.7 Confocal Raman spectroscopic system ......................................................... 19 


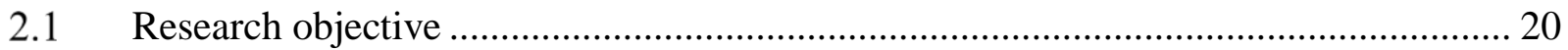

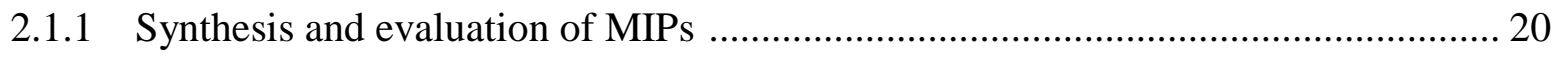

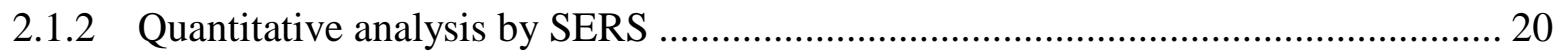

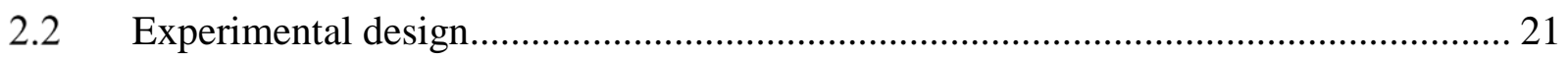

Chapter 3: Determination of thiabendazole in orange juice using a MIP-SERS

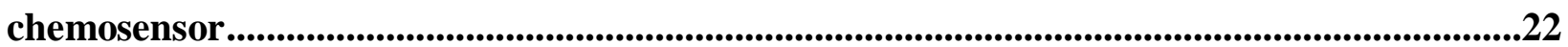

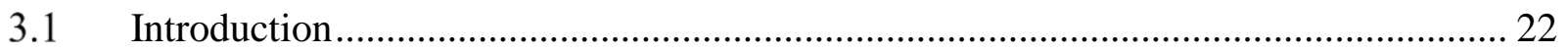

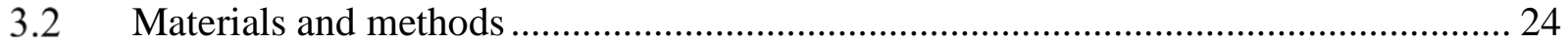

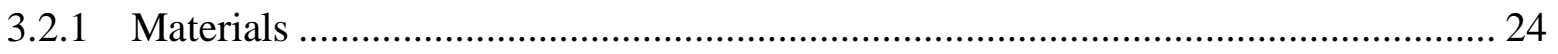

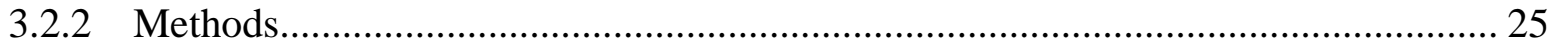

3.2.2.1 Synthesis of molecularly imprinted polymers (MIPs) …………………….... 25

3.2.2.2 Characterization of MIPs .......................................................................... 25

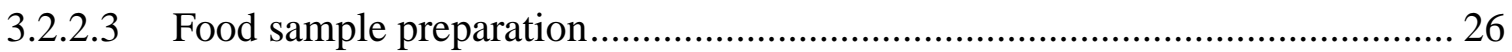

3.2.2.4 Molecularly imprinted solid phase extraction (MISPE) ................................. 26

3.2.2.5 High-performance liquid chromatography ..................................................... 27

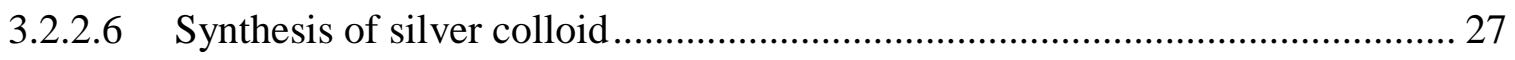

3.2.2.7 Scanning electron microscopy (SEM) ………............................................... 27

3.2.2.8 Raman spectral collection and multivariate statistical analysis ........................ 28

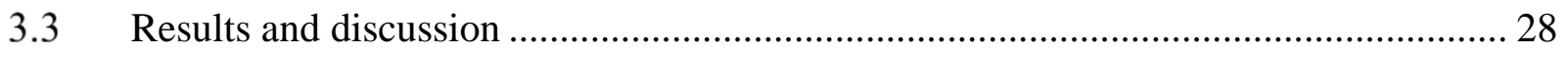

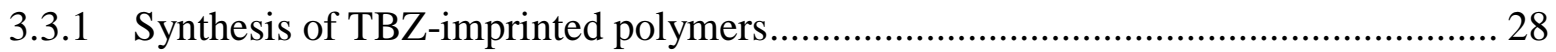

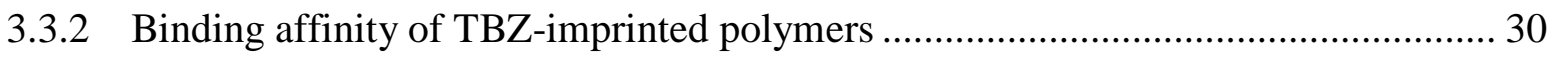

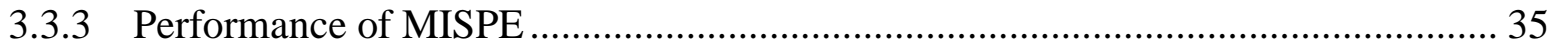


3.3.4 Quantitative analysis of TBZ in orange juice by SERS

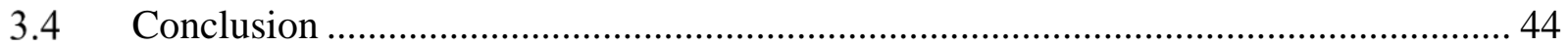

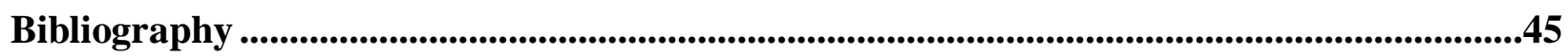

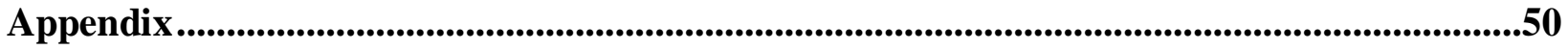




\section{List of Tables}

Table 1.1 Advantages and disadvantages of covalent and non-covalent imprinting. ${ }^{11}$............... 5

Table 3.1. The average recovery of thiabendazole (TBZ) in orange juice by molecularly imprinted solid phase extraction (MISPE) and non-imprinted solid phase extraction (NISPE) $(\mathrm{N}=3)$.

Table 3.2. Partial least squares regression (PLSR) models for the prediction of thiabendazole concentration in orange juice.

Table A. 1 Chemicals used in the MIPs synthesis process. 


\section{List of Figures}

Figure 1.1 Chemical structure of thiabendazole. ...................................................................... 1

Figure 1.2 The number of publications searched in PubMed within the field of molecular

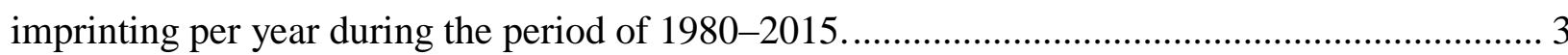

Figure 1.3 Schematic illustration of the imprinting process showing some of the interactions used in creating affinity in the binding site for the template. ${ }^{10}$ The interactions with functional motifs (a-d) of the template represent the following imprinting strategies. (a) Non-covalent H-bonding with methacrylic acid, acrylamide, etc.; (b) Reversible covalent interaction, such as a boronate ester; (c) Semi-covalent (sacrificial spacer) method; (d) Electrostatic interaction with an

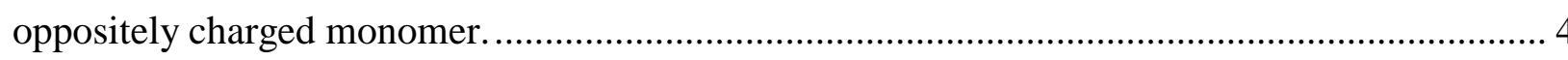

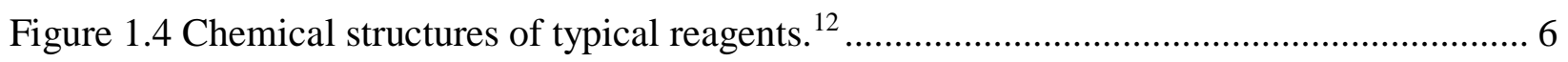

Figure 1.5 Diagram of Raman scattering and Rayleigh scattering illustrated by classical theory: compared to the incident electromagnetic radiation with frequency $v_{0}$, Stokes Raman with lower frequency $\left(v_{0}-v_{\text {vib }}\right)$, anti-Stokes Raman with higher frequency $\left(v_{0}+v_{\text {vib }}\right)$ and Rayleigh scattering

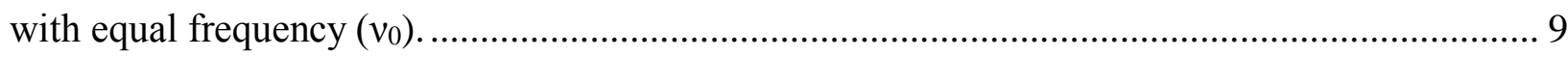

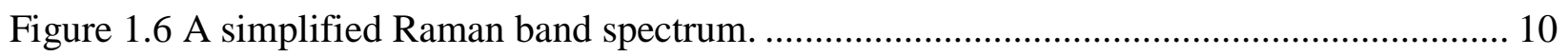

Figure 1.7 A Jablonski diagram illustrates quantum energy transitions for Rayleigh and Raman scattering.

Figure 1.8 Electromagnetic enhancement in SERS achieved in two steps. a) An overview of the working principle of EM enhancement. b) Step one: Incident electromagnetic wave drives a charge separation on sliver nano-particle and then induced a dipolar localized surface plasmon resonance (LSPR). c) Both the "incoming" and the "outgoing" fields are enhanced by elastic light scattering off the surface of nano-metal structure with dipolar LSPR ................................ 15 
Figure 1.9 Schematic illustration of (a) a confocal and (b) a conventional microscope coupled to a spectrometer. ${ }^{43}$ 19

Figure 2.1 Schematic illustration of MIPs-SERS chemosensor for the detection of thiabendazole in orange juice. MIPs: molecularly imprinted polymers; SERS: surface enhanced Raman spectroscopy. (TBZ: thiabendazole, MAA: methacrylic acid, DVB: divinylbenzene, CCD: charge-coupled device) 21

Figure 3.1 The synthesis process of MIPs towards TBZ. (a) Starting materials are listed as follow: template molecule (thiabendazole), functional monomer (methacrylic acid), and initiator (AIBN: 2, 2-azobisisobutyronitrile). (b) Proposed hydrogen bonding will form between a template molecule and functional monomers in the pre-polymerization stage. (c) Polymerization with cross-linking agents is carried out in the presence of the complex. (d) Subsequent removal of the template yields a binding site complementary to the template molecule. 30 Figure 3.2 The ratio of absorbance of supernatant in NIPs to that in MIPs towards $10 \mathrm{ppm} \mathrm{TBZ}$ after 3-h incubation as a function of the percentage of acetonitrile in the aqueous solution. 31 Figure 3.3 (a) Static binding isotherm of molecularly imprinted polymers (MIPs) and nonimprinted polymers (NIPs) towards thiabendazole; (b) Scatchard plot of MIPs and NIPs toward thiabendazole; (c) Kinetic binding isotherm of MIPs and NIPs (Ci: $10 \mathrm{mg} \mathrm{L}^{-1}$ ). Ci: initial concentrations of thiabendazole; Q: binding capacity. Error bars indicate standard deviation $(\mathrm{N}=$ $3)$.

Figure 3.4 The separation layers of orange juice after sample preparation. 35 Figure 3.5 Schematic illustration of molecularly imprinted solid phase extraction (MISPE) of TBZ in orange juice. 36

Figure 3.6 Representative scanning electron microscope image for sliver collide 38 
Figure 3.7 Representative spectral features of thiabendazole (TBZ) with (a) normal Raman of TBZ powder, (b) SERS of standard TBZ solution in methanol (50 $\left.\mathrm{mg} \mathrm{L}^{-1}\right)$, and (c) MISPE-SERS

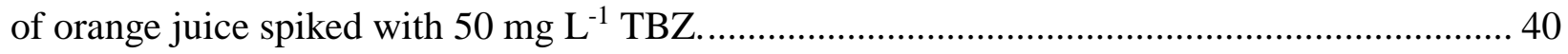
Figure 3.8 Schematic illustration of the orientation model of TBZ onto the surface of silver colloid. Left: The lone pair electrons on $\mathrm{S}$ and $\mathrm{N}$ atom result in a standing-up orientation of TBZ to the surface of Ag nanoparticles. Right: The $\pi$ ring electrons result in a planar coverage of the surface by TBZ.

Figure 3.9 Representative normal Raman spectral features with (a) thiabendazole powder, Representative MIPs-SERS spectra of orange juice spiked with different concentrations of thiabendazole: (b) $0 \mathrm{mg} \mathrm{L}^{-1}$, (c) $10 \mathrm{mg} \mathrm{L}^{-1}$, (d) $25 \mathrm{mg} \mathrm{L}^{-1}$, (e) $50 \mathrm{mg} \mathrm{L}^{-1}$, and (f) $75 \mathrm{mg} \mathrm{L}^{-1} \ldots \ldots . .41$ Figure 3.10 Two-dimensional principal component analysis for orange juice spiked with different concentrations of thiabendazole (TBZ). Principal component (PC)1 represents 86.3\% variances and PC2 represents 7\% variances. Letters from A to E denote orange juice spiked with $\mathrm{TBZ}$ at the concentration of $0,10,25,50$ and $75 \mathrm{mg} \mathrm{L}^{-1}$, respectively. ................................... 42

Figure 3.11 Partial least squares regression (PLSR) model predicts the actual concentrations of TBZ in orange juice.

Figure A. 1 Representative UV-absorbance spectrum of thiabendazole solutions with different conditions. a) $7.5 \mathrm{ppm}$ TBZ solution store under room temperature for 24 hours. c) $7.5 \mathrm{ppm}$ TBZ solution store at $4{ }^{\circ} \mathrm{C}$ for 24 hours. c) Compare absorbance spectrum between two conditions. d)

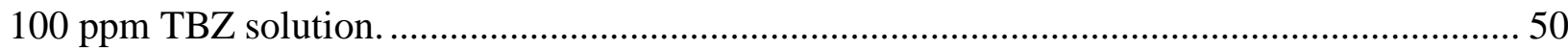

Figure A. 2 The UV absorbance of thiabendazole solution with different concentration. ............ 51 
Figure A. 3 Proposed mechanism for NIPs synthesis process. a) Initiation step: AIBN decomposed under heat and generate free radicals. b) Propagation step: Free radicals react with Methacrylic acid or the cross-linker DVB to propagate the chain further........ 


\section{List of Abbreviations}

AgNPs

AIBN

CCD

DAD

DMF

EGDMA

EM field

GC

HPLC

LC

LOD

LOQ

LSPR

MAA

MIPs

MISPE

MS

MW

NIPs

NISPE

PC

PCA

PPM

$\mathrm{Q}$ value

R2

SERS

SPE

SPR

UV silver nanoparticles

2,2'-azobis(isobutyronitrile)

charge coupled device

photodiode array detector

dimethylformamide

ethylene glycol dimethacrylate

electromagnetic field

gas chromatography

high performance liquid chromatography

liquid chromatography

limit of detection

limit of quantification

local surface plasmon resonance

methacrylic acid

molecularly imprinted polymers

molecularly imprinted solid phase extraction

mass spectrometry

molecular weight

non-imprinted polymers

non-imprinted solid phase extraction

principal component

principal component analysis

part per million

adsorption capacity value

coefficient of determination

surface enhanced Raman spectroscopy

solid phase extraction

surface plasmon resonance

ultraviolet 


\section{Acknowledgements}

I would like to show my gratitude to my co-supervisors: Dr. Xiaonan Lu and Dr. Ed Grant for their guidance and support from the initial to the final level enabled me to develop an understanding of the subject. Furthermore, I would like to thank my committee members: Dr. Keng Chou and Dr. Fleming for your time commitment and invaluable comments and suggestions throughout my study.

I thank all the Lu lab members in for the stimulating discussions, and for all the fun we have had in the last three years, especially Luyao Ma, Shaolong Feng and Yaxi Hu. Also, I want to offer my thanks to my best friend, Leixing Chen, and I treasure all the laughter we have shared in the last seven years.

Last but not the least, I offer my deepest thanks to my husband, Zeyu Han, and my beloved parents, Ailan Zhang and Yunling Feng, for all your love, care and supporting me spiritually throughout entire process. I will be grateful forever for your love. 


\section{Dedication}

To mom, who gave me endless love and support. 


\section{Chapter 1: Introduction}

\subsection{Thiabendazole pesticide residues in foods}

Thiabendazole (TBZ) is a systemic fungicide used as a post-harvest dip or spray on citrus fruit for preventing diseases derived from mold, blight, rot and stains (Figure 1.1). As a systemic fungicide, thiabendazole can penetrate through cuticles into the plant tissues within 6 hours; therefore, TBZ can easily remain in the food products as a pesticide residue. ${ }^{1}$ Health Canada has proposed the maximum residual limit (MRL) of thiabendazole in citrus fruit as ten parts per million (ppm). ${ }^{2}$

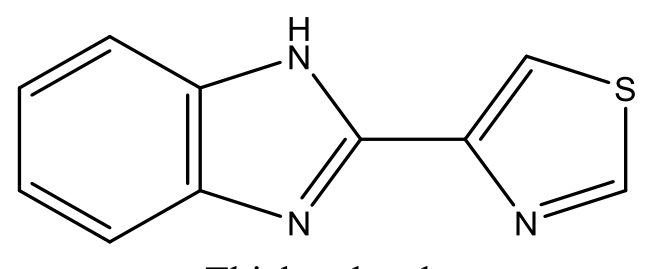

Thiabendazole

Figure 1.1 Chemical structure of thiabendazole.

The solubility of thiabendazole in water is $50 \mathrm{mg} / \mathrm{L}$ at $25^{\circ} \mathrm{C}$. The substance does not degrade under thermal processing conditions or upon exposure to light . The maximum of UV absorption is near $298 \mathrm{~nm}$. The polar surface area (PSA) of thiabendazole is $67 \AA^{2}$, where PSA refers to the sum over the surface area of all polar atoms, primarily oxygen and nitrogen inclusive of their attached hydrogen atoms. The PSA value is commonly used to evaluate the ability of a chemical compound (i.e. drug molecule) to permeate cells. A molecule with a PSA value less than $90 \AA^{2}$ can penetrate the blood-brain barrier. Based upon its small PSA value, thiabendazole presents a threat to the human central nervous system. 
The complexity of food matrices makes it challenging to rapidly determine trace levels of thiabendazole in food products. Traditional methods are laborious, time-consuming, and require the use of a large amount of solvent for the extraction of TBZ from food products. A specific recognition method is vital. Molecularly imprinted polymers (MIPs) will be introduced in the next section, which can offer a tailor-made receptor for a specific target molecule.

\subsection{History of molecularly imprinting technique}

Molecular imprinting originated from work in 1931 by the Russian scientist, M.V. Polyakov³ When he used sodium silicate solution with an ammonium carbonate as an initiator to prepare the silica gels, the prepared gels selectively absorbed certain solvent molecules. This research idea was then introduced into biological research by Linus Pauling in 1940. Pauling identified a way to form antibody by a self-assembly process of protein antibody around an antigen template. It took about thirty years for scientists to develop a clear method for molecular imprinting. In 1972, a paper written by Wuff and Sarhan described a covalent approach to molecular imprinting by using synthetic organic polymers ${ }^{4}$. Based upon the research achievement of Wuff and Sarhan, Shea and colleagues ${ }^{5,6}$, Neckers and colleagues ${ }^{7}$, and Mosbach and colleagues ${ }^{8}$ did further research related to molecular imprinting, and their work verified that the polymers with selective cavities could be synthesized. Since then, many researchers have become interested in this molecular imprinting technology with the aim of designing tailor-made polymers for specific compounds. A continuous growth in the published papers related to molecular imprinting is shown in Figure 1.2. A total of 5921 articles have been published in the recent twenty-five years. Among them, 275 articles combined molecular imprinting with solid phase extraction to separate and enrich analytes before detection. 


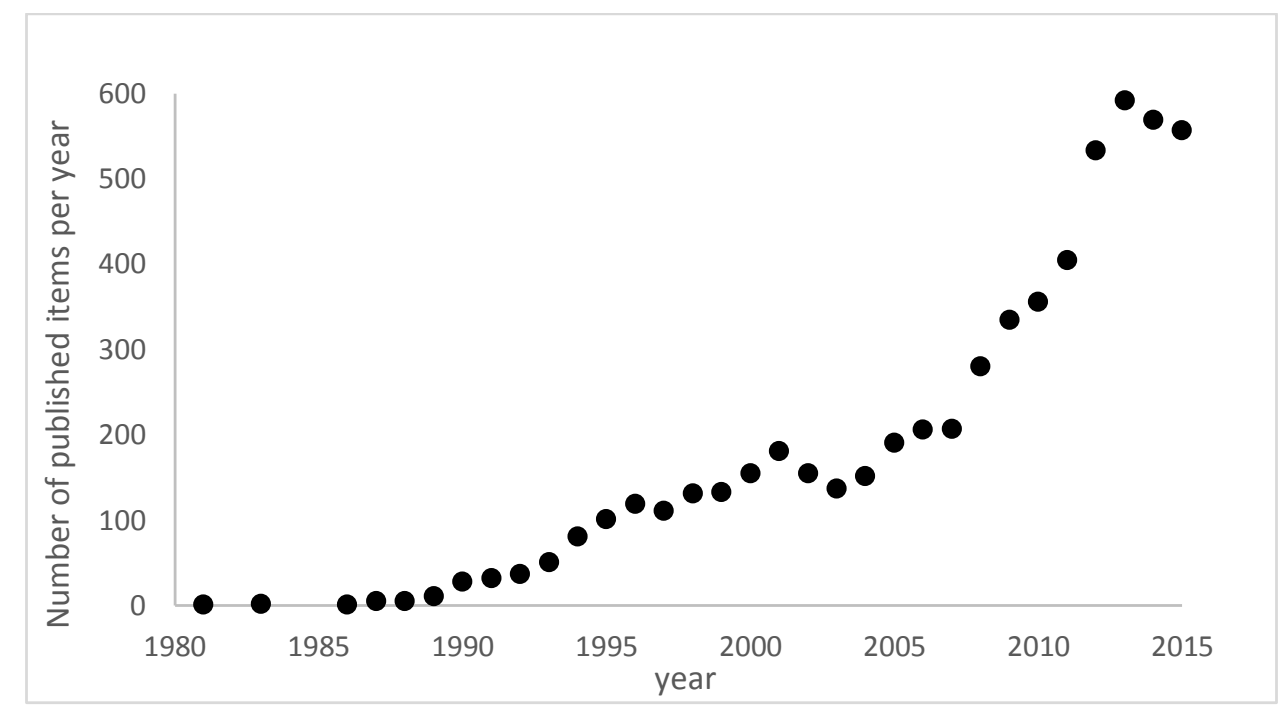

Figure 1.2 The number of publications searched in PubMed within the field of molecular imprinting per year during the period of 1980-2015.

\subsubsection{General mechanism of molecular imprinting}

Molecular imprinting is a technique to design a tailor-made receptor with specific binding sites complementary to a template molecule ${ }^{9}$. This tailor-made receptor is similar to an artificial "antibody" that can catch the target molecule (e.g., TBZ). The process of molecular imprinting can be summarized in three steps (Figure 1.3). Firstly, the templates are surrounded by functional monomers; they interact and combine to form a stable template-monomer complex through either covalent binding or non-covalent binding. This step is the fundamental part of a successful molecular recognition. Then, the complexes are positioned spatially and fixed by copolymerization with crosslinking agents. The last step removes the template from the polymer by washing with a solvent. The final adduct is a porous polymer with specific binding sites 
complementary to the template in size, shape and chemical functional group. This type of polymer is defined as a molecularly imprinted polymer (MIPs). In effect, the polymer has imprinted a target molecule in its memory, which now can specifically rebind the target. Therefore, MIPs can be considered as a tailor-made receptor, as it recognizes key features of analytes and can bind specific target molecules.
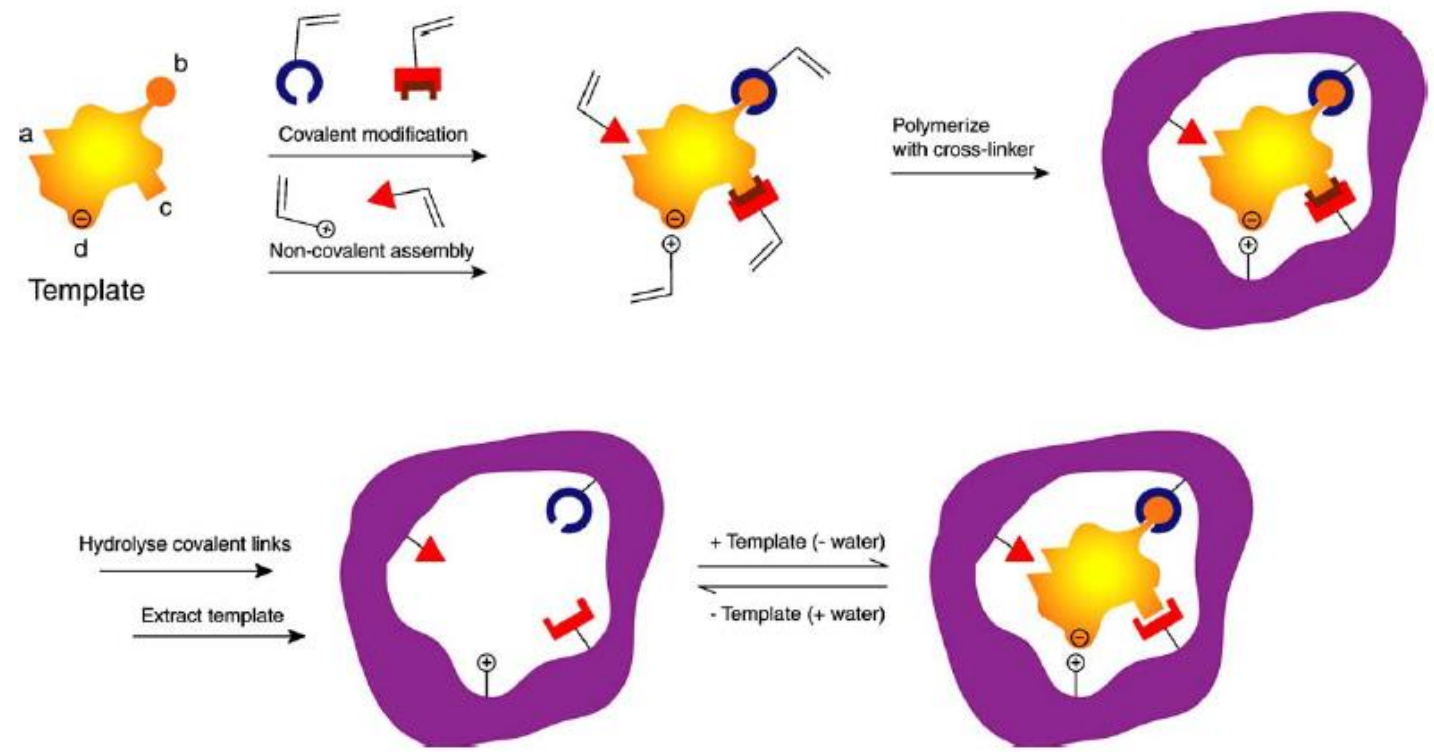

Figure 1.3 Schematic illustration of the imprinting process showing some of the interactions used in creating affinity in the binding site for the template. ${ }^{10}$ The interactions with functional motifs (a-d) of the template represent the following imprinting strategies. (a) Non-covalent H-bonding with methacrylic acid, acrylamide, etc.; (b) Reversible covalent interaction, such as a boronate ester; (c) Semi-covalent (sacrificial spacer) method; (d) Electrostatic interaction with an oppositely charged monomer.

As mentioned above, the type of imprinting depends upon the interaction between templates and monomers, either covalent bonding or non-covalent bonding. Briefly, it is easier to achieve noncovalent imprinting and apply this method to a variety of templates. However, the covalent 
imprinting will make the polymer more precise for the target molecule. Other characteristics are summarized in Table 1.1 by Komiyama and others ${ }^{11}$.

Table 1.1 Advantages and disadvantages of covalent and non-covalent imprinting. ${ }^{11}$

\begin{tabular}{lll}
\hline & Covalent & Non-covalent \\
\hline Synthesis of monomer- & Necessary & Unnecessary \\
template conjugate & & \\
\hline Polymerization conditions & Rather free & Restricted \\
\hline Removal of template after & Difficult & Easy \\
polymerization & & \\
\hline Guest-binding and guest- & Slow & Fast \\
release & & Less clear \\
\hline Structure of guest-binding & Clearer & \\
site & & \\
\hline
\end{tabular}

\subsection{Synthesis of MIPs}

In general, there are three types of synthesis mechanisms for polymerization in molecular imprinting: namely bulk polymerization, precipitation polymerization, and sol-gel polymerization. Before introducing different mechanisms, some basic reagents that most frequently used are listed in Figure 1.4. As mentioned above, three main types of reagents are needed: functional monomers, crosslinking agents, and radical initiators. 
Functional monomers<smiles>C=C(C)C(=O)O</smiles>

Methacrylic acid

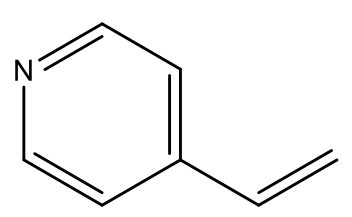

4-vinylpyridine

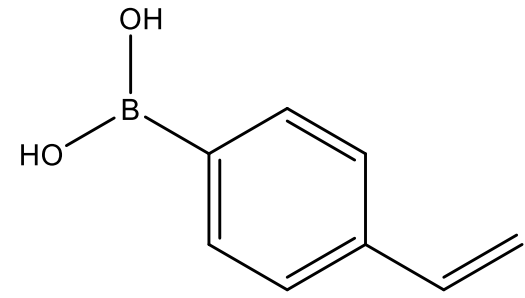

4-vinylbenzeneboronic acid

Crosslinking agents<smiles>C=C(C)C(=O)OCCOC(=O)C(=C)C</smiles>

Ethylene glycol dimetharylate EGDMA

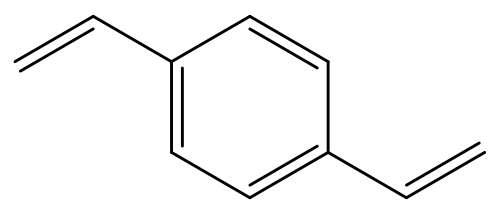

Divinylbenzene DVB

Radical initiators

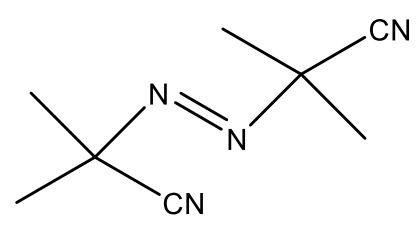

2,2'-azobis (isobutyronitrile) AIBN

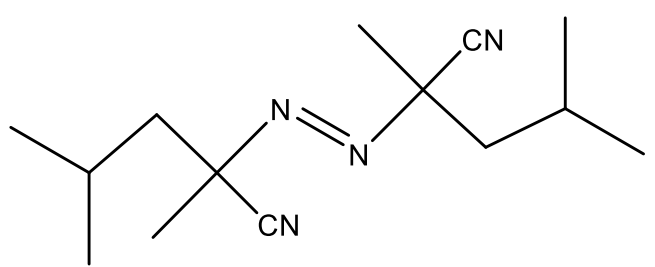

2,2'-azobis (2,4-dimethylvaleronitrile) ADVN

Figure 1.4 Chemical structures of typical reagents. ${ }^{12}$

Bulk polymerization can be initiated by using thermal decomposition of radical initiators. ${ }^{13}$ AIBN or ADVN are usually used as the initiator to produce radicals for promoting the polymerization. Bulk polymerization is very straightforward and economical, but the polymer formed will be in micro-size with a rough surface. This product form carries the template with 
less efficiency owing to cavities of lesser surface area. It is important to remove molecular oxygen from bulk polymerization mixtures because $\mathrm{O}_{2}$ combines with radicals, which reduces or stops the polymerization. To avoid oxygen, nitrogen gas or argon gas (inert gas) is often used to purge the solutions.

Ye and colleagues ${ }^{14}$ describe precipitation polymerization in an easily understandable way, the initial (linear and branched) aggregate into larger entities and phase-separate oligomer radicals incorporating template-functional monomer complex and crosslinker grow, they begin to interconnect with each other, from solution while absorbing more template-functional monomer complex and crosslinker. Then, the enlarged particles are formed to produce a highly cross-linked polymerization mixture.

Compared to bulk polymerization, sol-gel process can be carried out at a relatively lower temperature. The metal alkoxide solution will undergo hydrolysis in the liquid phase, which is then changed to gel during polymerization. In addition, the end product will have a higher purity and be more homogenous. There exists another different step for sol-gel polymerization from the other two reactions, which is adding precursor to aid in the formation of sol-gel. The precursor is similar to initiator that facilitates radical polymerization. The precursor will form a colloidal solution (sol) at first and then combine the functional monomers around it to form a gel. As described by Jennifer and coauthors ${ }^{15}$, metal alkoxide molecular is used as the precursors to produce a metal oxide during the reaction:

$$
\mathrm{nSi}(\mathrm{OR})_{4}+2 n \mathrm{H}_{2} \mathrm{O} \rightarrow n \mathrm{SiO}_{2}+4 n \mathrm{ROH}
$$

, where $\mathrm{R}$ is an alkyl group and the metal is silicon. 


\subsection{The theory of Raman scattering}

There are two prevailing theories to illustrate Raman scattering: one is the classical approach and the other one is the quantum mechanical theory. In the classical approach, the light is considered as electromagnetic radiation. When a beam of light shines on a molecule, an oscillating electric field produced by electromagnetic radiation will interact with the molecule through its polarizability. In classical theory, the ability of electron cloud of the molecule to interact with an electric field will determine the polarizability. This interaction creates an induced dipole moment in the molecule. The light scattered by this induced dipole includes both Rayleigh scattering and Raman scattering. The majority of the scattered light is elastically scattered (Figure 1.5), indicating that the frequency of scattered light will be the same as that of the incident light. This elastically scattering is called as Rayleigh scattering. ${ }^{16}$ Only a small amount of light is inelastically scattered, the Raman scattering, where the scattered radiation will shift in frequency from the incident radiation via gaining or losing vibrational energy in the molecule. Hence, there are two types of Raman shifts shown in Figure 1.5: one is known as an anti-Stokes shift that results in an increase in frequency and the other is called Stokes shift, which leads to the decrease in frequency. 


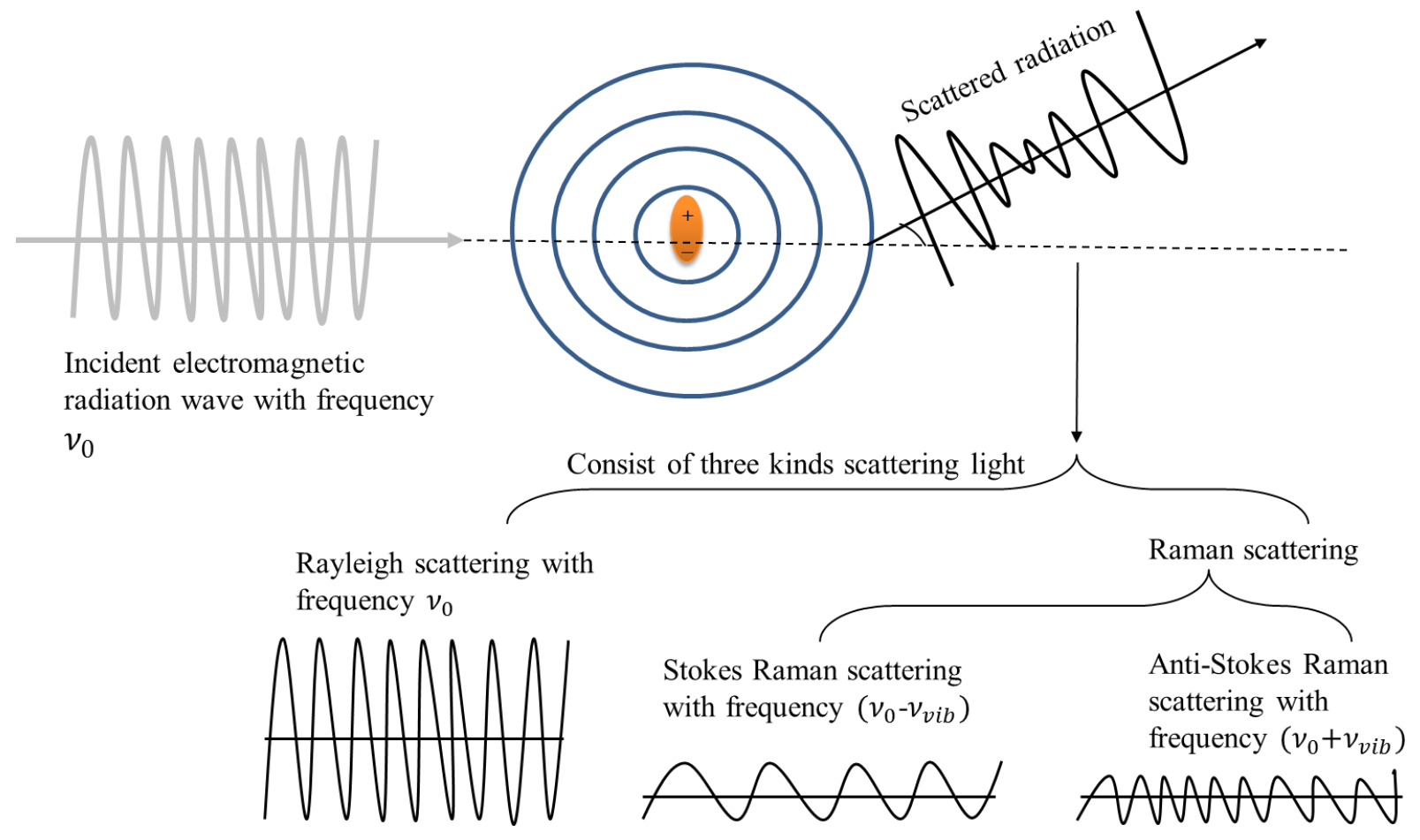

Figure 1.5 Diagram of Raman scattering and Rayleigh scattering illustrated by classical theory: compared to the incident electromagnetic radiation with frequency $v_{0}$, Stokes Raman with lower frequency $\left(v_{0}-v_{v i b}\right)$, antiStokes Raman with higher frequency $\left(v_{0}+v_{v i b}\right)$ and Rayleigh scattering with equal frequency $\left(v_{0}\right)$.

In a Raman spectrum, a beam of the laser (electromagnetic radiation) is focused on the sample, and then the intensity of Raman signal (scattered radiation) is measured as a function of its wavelength. Hence, the intensity is plotted as a function of the Raman wavenumber $\omega$ with units expressed in $\mathrm{cm}^{-1}$. This wavenumber is related to the difference between the scattered radiation and the incident light ${ }^{17}$ :

$$
\omega=\overline{v_{m}}-\overline{v_{0}}=\frac{v_{m}}{c}-\frac{v_{0}}{c}
$$

In this equation, $v_{\mathrm{m}}$ stands for the frequency of the scattered radiation, $v_{0}$ represents the frequency of the incident radiation, $\mathrm{c}$ is the speed of light, $\omega$ is the wavenumber and expressed in 
$\mathrm{cm}^{-1}$. Therefore, Stokes shift corresponds to positive wavenumbers, and anti-Stokes shift corresponds to negative wavenumbers (shown in Figure 1.6).

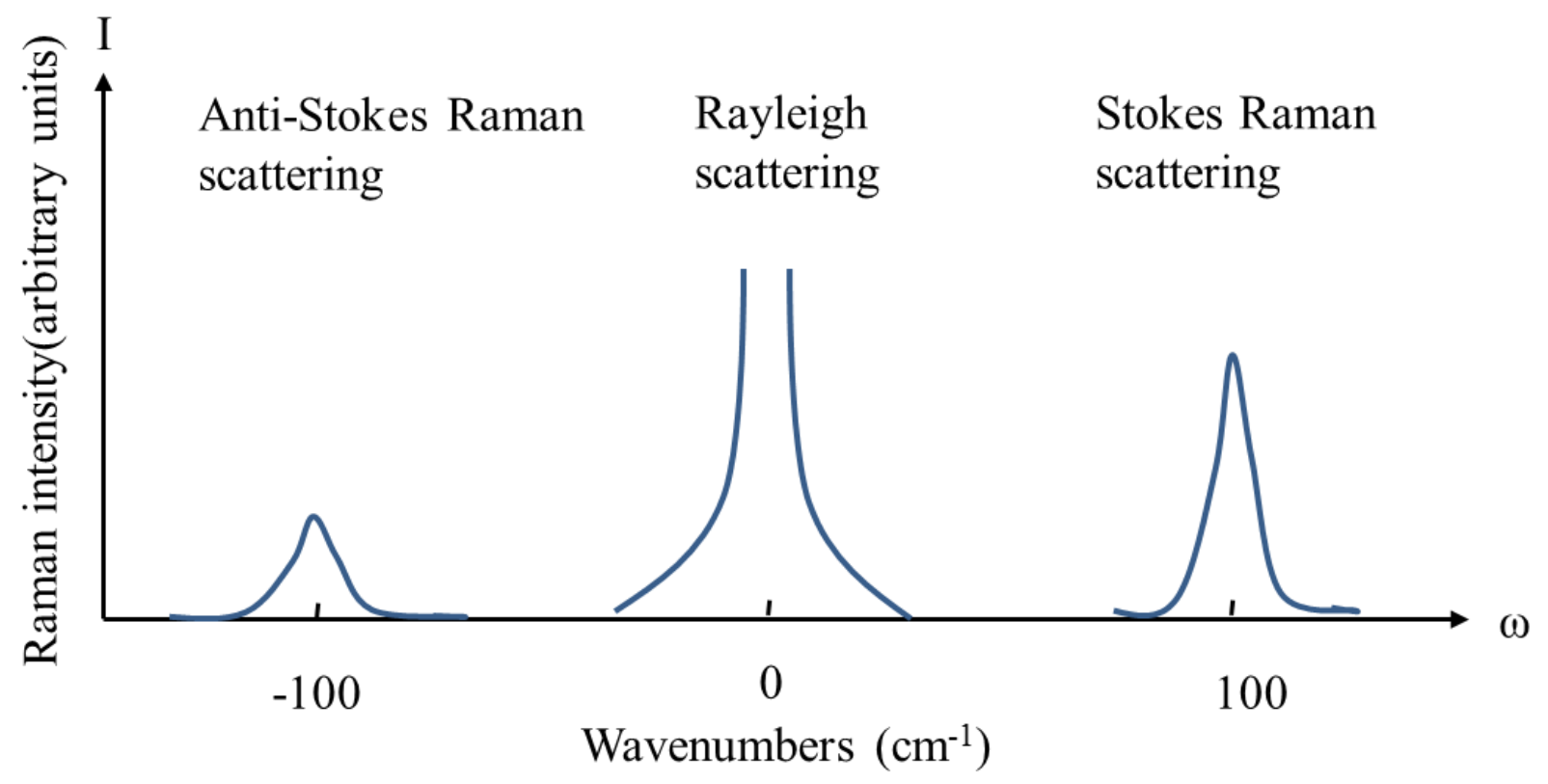

Figure 1.6 A simplified Raman band spectrum.

When we consider the quantum mechanical theory, the light is regarded as a photon which strikes the molecule and inelastically scatters. A Jablonski diagram is shown in Figure 1.7, which explains quantum energy transitions for Rayleigh and Raman scattering. The incident photon excites the molecule from the zeroth ground electronic state into a virtual energy state, resulting in three potential outcomes. The left part of the figure shows Stokes-shifted Raman scattering. Specifically, the molecule will relax to a vibrational state and the scattered photon has less energy than the incident photon. The middle part is an elastic process, referred to as Rayleigh scattering. The molecule goes back to the zeroth ground state, and the scattered photon has the equal energy to that of the incident photon. The right part shows anti-Stokes Raman scattering 
where the molecule starts with in an excited vibrational state. A Raman transition that ends in the initial ground state emits a photon with more energy than the incident photon. At room temperature, most molecules occupy the vibrational ground state and there is a much higher probability that a photon will be Stokes scattered. Therefore, most Raman spectroscopy only considers Stokes shifted light with positive wavenumbers.

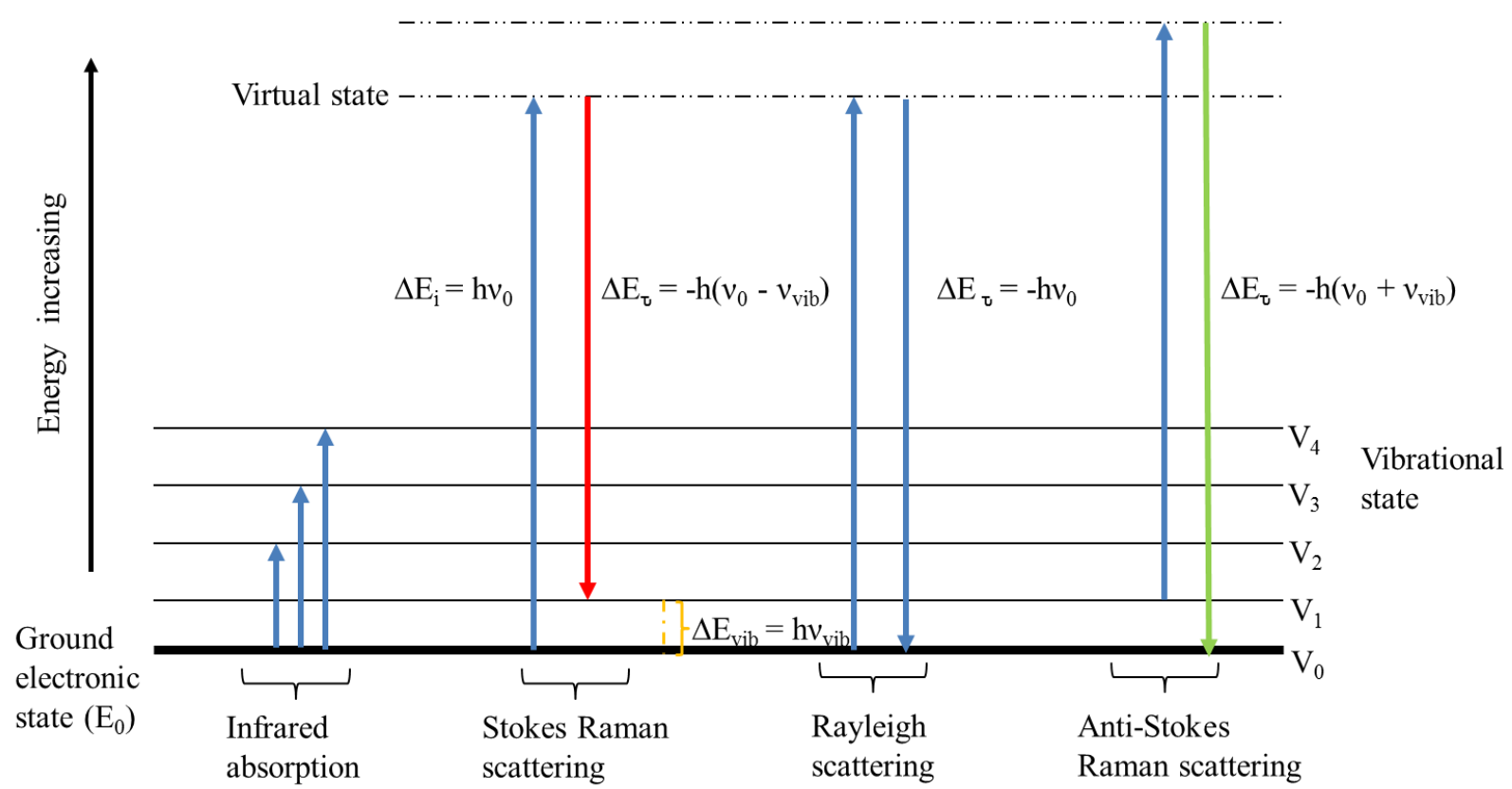

Figure 1.7 A Jablonski diagram illustrates quantum energy transitions for Rayleigh and Raman scattering.

Raman spectroscopy is a type of vibrational spectroscopy that can yield both structure and chemical information about a molecule. The Raman bands arise from the change in polarizability due to an interaction between light and the molecule., The bond between two similar or identical atoms (e.g., $\mathrm{C}=\mathrm{C}$ bond) is more active than a weakly polarizable bond (e.g., $\mathrm{O}-\mathrm{H}$ bond).

Therefore, water has an extremely weak Raman scattering signal and Raman spectroscopy can detect and discriminate various chemicals and food samples in an aqueous condition. However, 
the intensity of Raman signal is many orders weaker than the fluorescence emission, making the detection of trace levels of compounds very challenging.

\subsection{Enhancement of Raman signal}

Several principles can be used to enhance the inherently weak Raman scattering signal. Among those principles, the two most important ones are resonance enhanced Raman spectroscopy (RR) and surface-enhanced Raman spectroscopy (SERS). More details about these two approaches are illustrated in the following sections.

\subsubsection{Resonance Raman (RR) spectroscopy}

If the wavelength of the excitation laser beam overlaps with the electronic absorption band of the molecule, the resonance Raman enhancement will arise. The resonant excitation of electronic transitions in molecules is called as resonance Raman (RR) scattering. Compared to the non-

resonant excitation, the enhancement factor of resonance Raman can reach up to $10^{6}{ }^{18}$ In addition, resonance Raman spectroscopy can also be applied to enhance the sensitivity for sitespecific molecules (i.e. aromatic amino acid residues, nucleotides, and heme groups). ${ }^{19}$ However, the limitation is that researchers can only focus on a specific group of molecules, where the signal of molecule can be enhanced by resonance effect, only if the Raman scattering of vibrational modes are close to or around the excited state. 


\subsubsection{Surface-enhanced Raman spectroscopy (SERS)}

Surface-enhanced Raman Scattering (SERS) is a technique that enhances the intensity of Raman signals with increasing orders of magnitude. Enhancement factors can be as high as $10^{11}$, which are sufficient to allow even single molecule detection using Raman spectroscopy. ${ }^{20}$ To achieve this, the noble metal (e.g., silver and gold) nanostructures as SERS substrates will generate a localized surface plasmon resonance, inducing an electromagnetic field. Target analytes approaching the surface of the substrate show significantly enhanced Raman cross-sections, leading to a lower limit of detection to theoretically single molecule level. Currently, there are two factors used to explained the SERS effect, namely chemical enhancement and electromagnetic enhancement, where the second factor is considered as the virtual one. The following two parts illustrate the working principle of these two factors.

\subsubsection{Chemical enhancement (CE)}

The chemical enhancement factor contributes much less than the electromagnetic factor, and the $\mathrm{CE}$ factor is no more than $100 .^{21}$ As a surface-effect, the molecules in the vicinity of the metallic surface are analyzed. The interactions between the absorbed molecules and the metallic surface arise the chemical enhancement. These interactions can be categorized as physisorption or chemisorption effects. Physisorption includes all of the weak interactions, such as electrostatic interactions and Van der Waals interactions. On the other hand, chemisorption occurs when a chemical bond is formed between the substrate and the analyte molecule. In consequence, these bonds may cause some band shifts or a relatively weak band. Chemisorption or physisorption is more similar to an auto-preconcentration effect, which tries to trap analytes as much as possible in the vicinity of the surface. Besides those interactions, the orientation effect is also important, 
especially for anisotropic molecules, because the Raman effect is orientation dependent. Thus, orientation will enhance the signal only if the molecules are well aligned. The molecules sometimes have a favor orientation due to the interaction with the substrate. For example, the benzene ring is more likely to be parallel to the surface of the substrate, thus the $\pi$-electrons on the ring can interact sufficiently with the metallic surface. Furthermore, charge transfer is the dominant contribution to the chemical enhancement. Charge transfers between the metal and the analyte molecule. Firstly, a photon excites an electron into a hot electron state, and then the hot electron is transferred to a virtual state. The hot electron is then transferred back to the metal and at last returned the electron to its initial state.

\subsubsection{Electromagnetic field enhancement (EM)}

As aforementioned, the intensity of the measured Raman signal is increased along with the increasing strength of the oscillating electromagnetic field, or in another term, the intensity of the laser light. As shown in Figure 1.8 a, by analogy to a nano-sized antenna, the metallic particle (SERS substrate) will pick up the signal from the incident laser light and then emit the signal as an amplifier. The signal is finally picked up by the analyte and transformed into an electrical signal. 


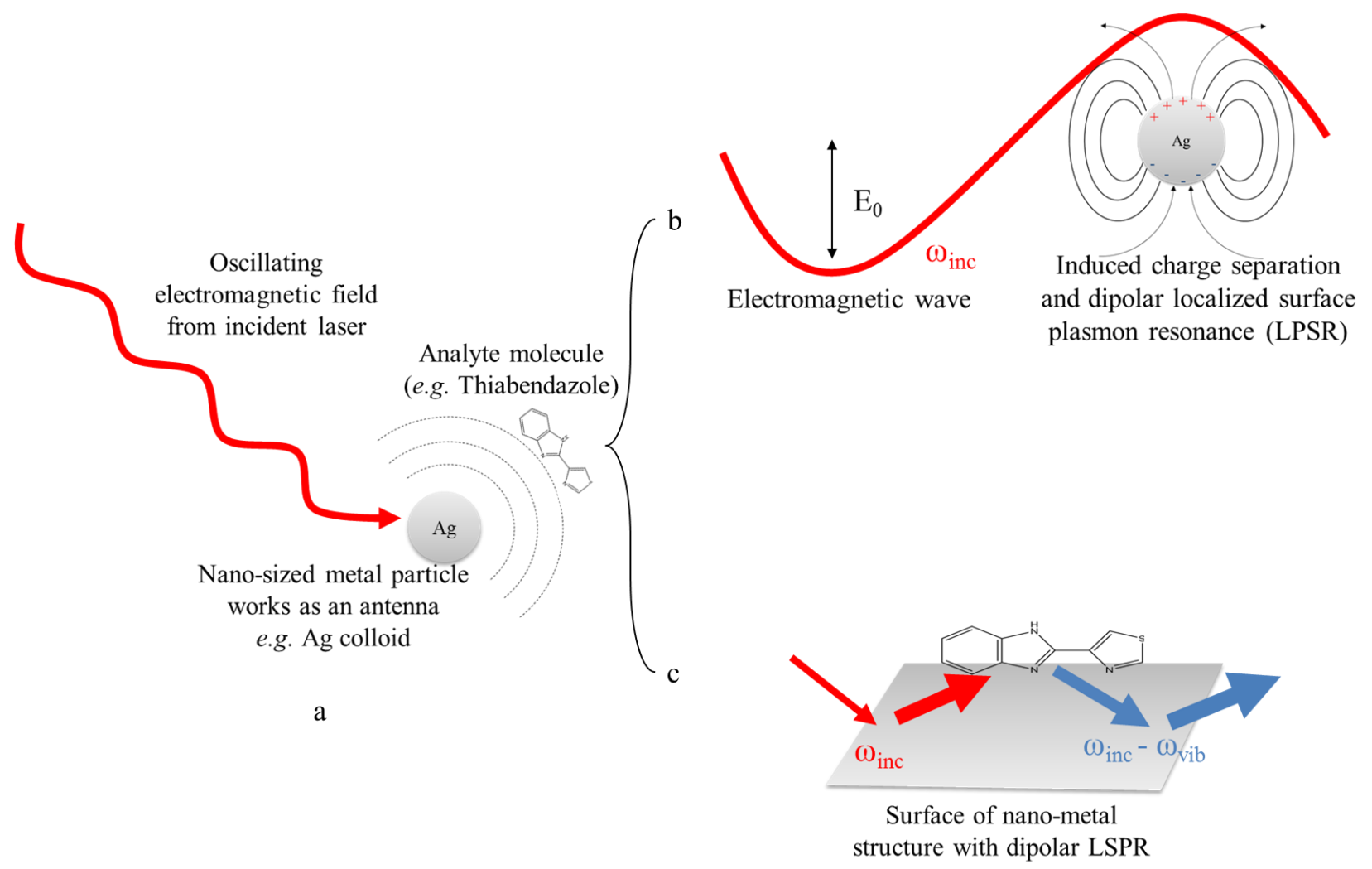

Figure 1.8 Electromagnetic enhancement in SERS achieved in two steps. a) An overview of the working principle of EM enhancement. b) Step one: Incident electromagnetic wave drives a charge separation on sliver nano-particle and then induced a dipolar localized surface plasmon resonance (LSPR). c) Both the "incoming" and the "outgoing" fields are enhanced by elastic light scattering off the surface of nano-metal structure with dipolar LSPR.

Therefore, we need to understand the interaction between the laser light and molecule and the interaction between the metallic particles. In Figure $1.8 \mathrm{~b}$, a laser beam or an electromagnetic wave resonantly excites the delocalized electrons in metal. According to resonant excitation, the oscillating electric field of incident radiation forces the conduction electrons to oscillate resonantly. Then, the resonant oscillation drives a charge separation in the metallic sphere, e.g. silver nanoparticle shows in Figure 1.8. This type of resonance is defined as dipolar localized 
surface plasma resonance (LSPR). The equation for the magnitude of the induced dipole $\mu_{\text {ind }}$ is shown as follow:

$$
\mu_{\text {ind }}=\alpha_{\text {metal }} E_{0}\left(\omega_{\text {inc }}\right)
$$

, where $\alpha_{\text {metal }}, \mathrm{E}_{0}\left(\omega_{\text {inc }}\right)$ represents the polarizability of the metal sphere and the incident electric field strength, respectively. $\omega_{\text {inc }}$ stands for the angular frequency of the electromagnetic wave. The sign of the localized induced dipole changed periodically and coincided with the angular frequency of the incident electromagnetic wave.

Figure $1.8 \mathrm{~b}$ explains the interaction between the laser and metallic particle. This interaction excites an emission from the substrate. In the meanwhile, an increased local electric field $E_{\text {loc }}\left(\omega_{\text {inc }}\right)$ is generated close to the metallic surface. Now we should consider the interaction between the generated local electric field $\mathrm{C}$ and the analyte attached on or close to the surface of the metallic sphere (in Figure $1.8 \mathrm{c}$ ). In contrast to the induced dipole $\mu_{\text {ind }}$ for metal, the equation for the magnitude of that for a molecule is shown as follow:

$$
\mu_{\text {ind }}=\alpha_{\text {molecule }} E_{\text {loc }}\left(\omega_{\text {inc }}\right)
$$

, where $\alpha_{\text {molecule }}, E_{\text {loc }}\left(\omega_{\text {inc }}\right)$ represents electronic polarizability of the molecule and the local incident electric field strength, respectively. In section 1.3, we use quantum mechanics to explain the Raman effect. Here, the local incident electric field $\mathrm{E}_{\text {loc }}\left(\omega_{\text {inc }}\right)$ is determined by a vibrating molecule with angular frequency $\omega_{\text {vib. }}$ It will generate three dipole components $\mu_{\text {ind }}\left(\omega_{\text {inc }}, \omega_{\text {inc- }}\right.$ $\omega_{\mathrm{vib}}$, and $\left.\omega_{\mathrm{inc}}+\omega_{\mathrm{vib}}\right)$ derived from Rayleigh, Stokes Raman, and anti-Stokes Raman scattering, respectively. The Stokes Raman scattering (the blue light) with angular frequency ( $\omega_{\text {inc- }} \omega_{\text {vib }}$ ) takes the dominant place for vibrations under selection rules. In Figure $1.8 \mathrm{c}$, the enhancement 
caused by elastic light scattering off the dipolar LSPR-supporting metallic surface works well for both the "incoming field" ( $\omega_{\text {inc }}$, red) and the "outgoing field" ( $\omega_{\text {inc }}-\omega_{\text {vib }}$, blue $)$. The SERS intensity can be expressed in the following equation:

$$
I_{S E R S}=I_{i n c}\left(\omega_{i n c}\right) I\left(\omega_{i n c}-\omega_{v i b}\right)=\left|E_{i n c} \omega_{i n c}\right|^{2}\left|E\left(\omega_{i n c}-\omega_{v i b}\right)\right|^{2}
$$

The optimal ISERS will occur when both incoming and outgoing fields are enhanced by the dipolar LSPR induced around the nano-sized metal. When incident laser band is close to the Stokes Raman scattering for a particular vibration Raman band, an approximation of ISERS can be used and expressed as $I_{S E R S} \approx\left|\mathrm{E}\left(\omega_{\text {inc }}\right)\right|^{4}$. Furthermore, SERS effect is distance dependent, and the adsorbate is not required to attach onto the surface directly. The electric field strength of a dipolar radiation is shown in the equation: $E_{(r)} \approx r^{-3}$, where $r$ is the distance form surface to adsorbate. The distance dependence of SERS intensity, given $\mathrm{E}^{4}$ enhancement, can be expressed as $I_{S E R S} \approx r^{-12}$.

Overall, the EM enhancement is a purely physical effect, which involves with the laser and SERS substrates. Compared to chemical enhancement, the EM enhancement contributes more and dominants the overall enhancement factor.

\subsection{Noise in Raman spectroscopy}

Noise always exists in a spectroscopic technique, and the term "noise" is defined as all those signals detected that do not contain any relevant information. Noise will severely affect the precision of obtained signals. In Raman spectroscopy, noise originates from three sources: the sample $\left(\sigma_{\mathrm{s}}\right)$, the Raman instrument $\left(\sigma_{\mathrm{i}}\right)$ and the signal-processing step $\left(\sigma_{\mathrm{p}}\right)$. The total noise level $(\sigma)$ involves with all three factors, and can be expressed as follows: 


$$
\sigma=\sqrt{\left(\sigma_{s}^{2}+\sigma_{i}^{2}+\sigma_{p}^{2}\right)}
$$

Typical noise sources include fluorescence, spikes and background noise. Spikes, also known as "ghost peaks," are caused by cosmic rays accidentally hitting the detector. The ghost peak is usually very sharp without reproducibility.

The noise that arises from the sample $\left(\sigma_{\mathrm{s}}\right)$ is fundamentally given by:

$$
\sigma_{S}=\sqrt{S}
$$

, where $\mathrm{S}$ stands for signal intensity, and this type of noise $\left(\sigma_{\mathrm{s}}\right)$ is recognized as inherent shot noise. For dispersive spectrometer, we have $S \propto$ the time measured. Therefore, $\sigma_{\mathrm{s}}$ is proportional to the square root of the time. Compared to the signal of analyte peaks, the noise peaks show up at random position. Therefore, the accumulation rate of noise signal is much lower than that of analyte signal, leading to a much smaller increasing in the intensity of the overall noise. For an FT-instrument, noise is proportional to the square root of the number of the scans. The larger the number of scans, the longer time required. As a consequence, the quality of a signal depends on the integration time. The optimal signal-to-noise ratio can reach by measuring for a longer time.

Every component of a Raman instrument contributes to a spectrum. The detector itself can be a source of noise. A detector generates dark signal due to thermal emission. Cooling down the detector can easily reduce the intensity of this signal. A laser with fluctuating output intensity causes variations in scattered light that can appear as noise. The confocal Raman system is used to minimize this noise, as discussed in the next section. 


\subsection{Confocal Raman spectroscopic system}

The first confocal microscope was constructed and patented by Minsky in $1957 .{ }^{22}$ Unlike the conventional microscope, a confocal microscope has the advantage of eliminating out-of-focus glare, and the ability to collect serial optical sections from thick specimens. The principle of a confocal microscope is to prevent all the scattered light from entering the microscope objective in the first instance by setting a pinhole aperture in front of the light source (Figure 1.9). By eliminating the scattered light from all other parts of the sample, the total amount of scattered light is then reduced by a few orders of magnitude without affecting the focal brightness. Therefore, the image quality is improved.

Compared with the conventional analytical techniques, the power of confocal spectral measurement in Raman spectroscopy enables a better differentiation of the sample from the ambient background. Thus, the advantages of high spatial resolution, clear image quality, etc. can be obtained.

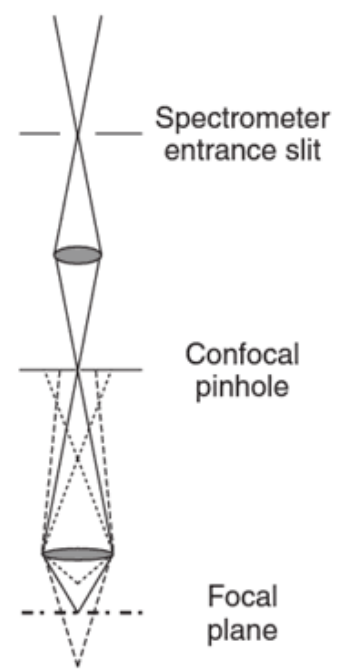

(a)

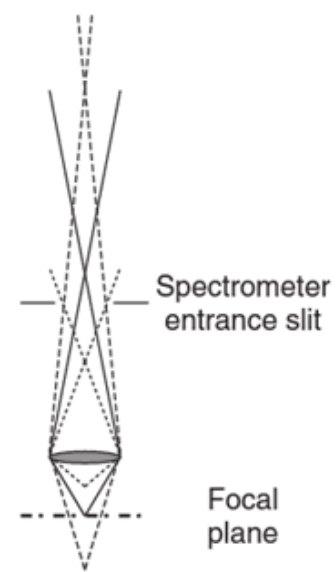

(b)

Figure 1.9 Schematic illustration of (a) a confocal and (b) a conventional microscope coupled to a spectrometer. $^{43}$ 


\section{Chapter 2: Research objectives and experimental design}

\subsection{Research objective}

The major objective of this thesis project was to develop an MISPE-SERS chemosensor for rapid determination of trace thiabendazole in orange juice with MIPs serving as SPE element and silver colloid as an SERS-active substrate for sensitive detection and quantification.

\subsubsection{Synthesis and evaluation of MIPs}

1) To evaluate the binding capacity of the synthesized MIPs, I conducted static adsorption and kinetic adsorption tests for both MIPs and NIPs.

2) To remove large molecules (e.g., polysaccharides, lipids, etc.) from orange juice, salting-out liquid-liquid partitioning method was used as a pretreatment step to the sample.

3) Recovery tests were carried out to validate the efficiency of MISPE column.

\subsubsection{Quantitative analysis by SERS}

1) Ag colloid was synthesized and applied as an SESR-active substrate to detect trace amount of the target analyte (TBZ).

2) To quantitatively determine TBZ in orange juice (e.g., calculate LOD), PCA model and PLSR model was developed. 


\subsection{Experimental design}

A detailed schematic illustration of MISPE-SERS chemosensor for the detection of thiabendazole in orange juice is shown in Figure 2.1. In brief, TBZ-imprinted MIPs was synthesized by precipitation polymerization, followed by removal of template via Soxhlet extraction. Adsorption test was then performed to validate the specific binding of MIPs towards TBZ. After that, synthesized MIPs were packed into the SPE cartridge as tailor-made sorbents for the separation and enrichment of TBZ from orange juice. The silver colloid was prepared and functioned as an SERS-active substrate to enhance signal intensity derived from the eluted TBZ. Various chemometric models, including PCA and PLSR, were then established to analyze the collected SERS signals.

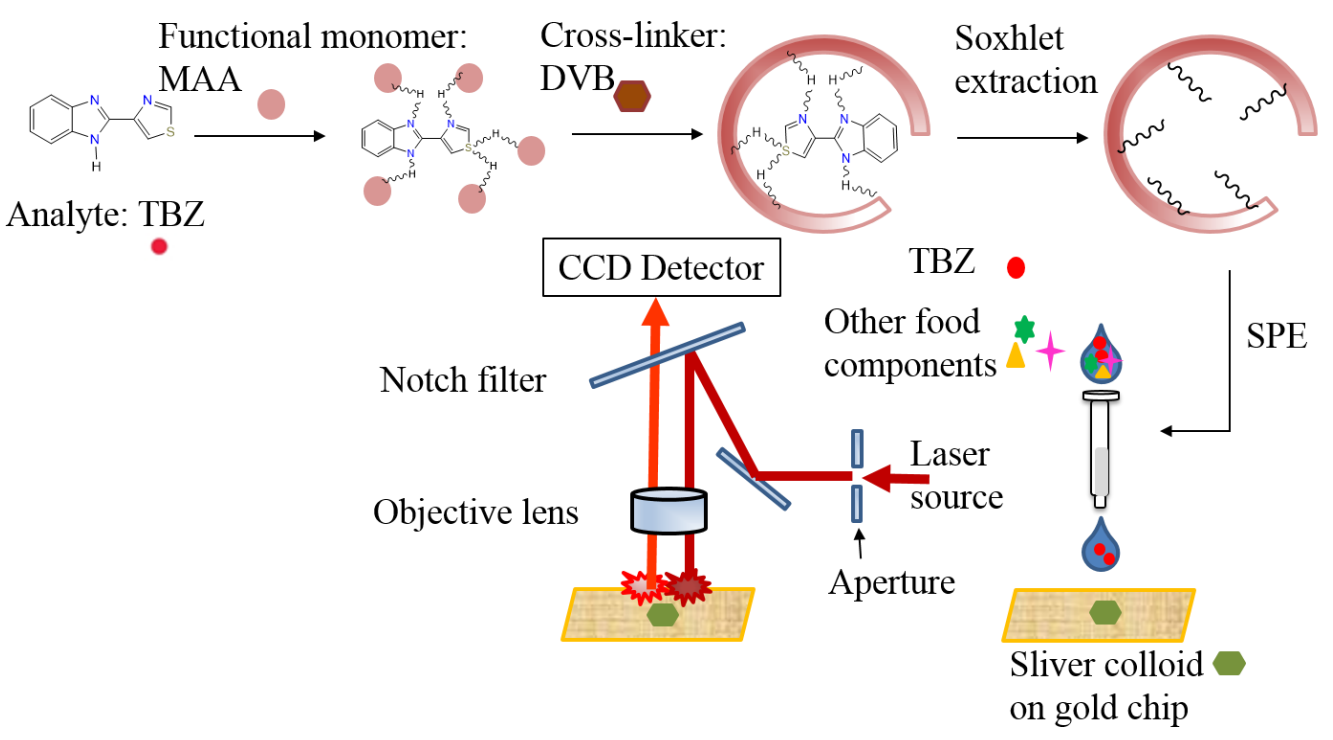

Figure 2.1 Schematic illustration of MIPs-SERS chemosensor for the detection of thiabendazole in orange juice. MIPs: molecularly imprinted polymers; SERS: surface enhanced Raman spectroscopy. (TBZ: thiabendazole, MAA: methacrylic acid, DVB: divinylbenzene, CCD: charge-coupled device) 


\section{Chapter 3: Determination of thiabendazole in orange juice using a MIP-SERS chemosensor}

\subsection{Introduction}

Thiabendazole (TBZ), a benzimidazole compound, is a commonly used postharvest pesticide to control diseases of citrus fruits caused by fungi (e.g., mold and rot). As a systemic fungicide, thiabendazole can penetrate through cuticles into the plant tissues within 6 hours; therefore, TBZ can easily remain in the food products as a pesticide residue ${ }^{1}$. TBZ can trigger cell death by apoptosis of hepatocytes, and it is detrimental to human health as it may cause severe liver damage $^{23}$. Pest Management Regulatory Agency (PMRA) of Health Canada has proposed the maximum residual limit (MRL) of thiabendazole in citrus fruit as 10 parts per million (ppm).

European Food Safety Authority (EFSA) has enacted a standard protocol for the determination of TBZ in orange juice. This multi-residual QuEChERS protocol in combination with liquid chromatography-tandem mass spectrometry (LC-MS/MS) method has been validated for the identification of TBZ with a LOD of $0.01 \mathrm{ppm}^{24}$. The QuEChERS acronym stands for Quick Easy Cheap Effective Rugged and Safe, which is an analytical procedure immensely simplifies the analysis of residues in sample. However, this method is laborious, time-consuming, and requires the use of a massive solvent for the extraction of TBZ from food products. Another method to determine TBZ residual in foods involves solid-phase extraction (SPE) for cleanup of food matrices, followed by high-performance liquid chromatography (HPLC)-photodiode array

UV detection ${ }^{25}$. This HPLC method requires less processing time, but leads to a higher LOD 
than LC-MS/MS method. An alternative method is thus required for rapid, accurate, and sensitive determination of TBZ in agri-foods in a high-throughput manner.

As a selective sorbent used in SPE, molecularly imprinted polymers (MIPs) has been recognized as a useful technique for specific and sensitive analysis of trace amount residuals in foods ${ }^{26}$. In the traditional SPE, the solid extraction phase with affinity to the target analyte can retain and concentrate the target compounds in a complex sample matrix, such as a food product ${ }^{27}$. However, this sorbent is not entirely peculiar to an analyte, leading to a relatively small recovery $(<90 \%)$. In this thesis project, MIPs are prepared by self-assembly protocol via copolymerization of functional monomer and cross-linker in the presence of the target molecule, called "template" 28. Three-dimensional binding cavities will remain in the polymeric matrix after the removal of template molecules. The cavity is complementary to the size, shape and chemical functionality of the template molecule. Thus, MIPs can be applied as specific sorbents in SPE to selectively separate and subsequently enrich the target molecules, resulting in a low LOD.

Surface-enhanced Raman spectroscopy (SERS) is a rapid, sensitive, and non-destructive detection technique that can be used as a reliable tool for food safety risk assessment ${ }^{29}$. Compared to the conventional Raman spectroscopy, SERS can enhance the intrinsically weak Raman scattering signal by several orders of magnitudes with the electromagnetic enhancement as the dominated factor ${ }^{21}$. The amplification of Raman scattering signal derives from the electromagnetic interaction between light and noble metallic nanostructures under the localized surface plasmon resonances (LSPR). It requires the analyte molecule to be attached on or in 
proximity (within $10 \mathrm{~nm}$ ) to the surface of metals ${ }^{20}$. The metallic nanostructures are usually made of silver or gold and called "SERS-active substrates" 30.

The aims of this thesis project are to synthesize MIPs with specific affinity to TBZ and use TBZMIPs as the sorbents in SPE to separate and enrich TBZ from orange juice. By preparing silver colloids as the SERS-active substrate, determination of thiabendazole in orange juice can be achieved by this MISPE-SERS chemosensor. Previously, Turiel and coauthors used TBZ-MIPs as coating outside on a stir ${ }^{31}$, a fiber ${ }^{32}$, or a frit for $\mathrm{SPE}^{33}$ to achieve separation of target analytes. In the current project, we for the first time fabricated a MIPs-SESR chemosensor for rapid determination of TBZ in orange juice.

\subsection{Materials and methods}

\subsubsection{Materials}

Methacrylic acid (MAA), divinylbenzene (DVB), 2,2'-azobis(isobutyronitrile) (AIBN), TBZ, silver nitrite, and trisodium citrate were purchased from Sigma-Aldrich (St. Louis, MO, USA). Methanol (HPLC grade), acetonitrile (HPLC grade), toluene (HPLC grade), and acetic acid were obtained from Thermo Fisher Scientific (Toronto, ON, Canada). Orange juice samples were purchased from several local grocery stores in Vancouver. TBZ stock solution $\left(0.5 \mathrm{mmol} \mathrm{L}{ }^{-1}\right)$ was prepared in $20 \%$ acetonitrile, stored at room temperature, and diluted with $20 \%$ acetonitrile for further use as working solution. The porogen solvent was made of acetonitrile/toluene $(3: 1$, v/v). 


\subsubsection{Methods}

\subsubsection{Synthesis of molecularly imprinted polymers (MIPs)}

The synthesis of MIPs was followed the procedure by Turiel and others with modification ${ }^{34}$. TBZ $(0.68 \mathrm{mmol})$ and methacrylic acid $(2.72 \mathrm{mmol})$ were added into $50 \mathrm{~mL}$ porogen solvent and sonicated for $15 \mathrm{~min}$, followed by addition of divinylbenzene $(13.6 \mathrm{mmol})$ and AIBN (1 mmol) into the mixture and degassed with nitrogen for $15 \mathrm{~min}$. This mixture reacted for 24 hours in a water bath with magnetic stir at $60^{\circ} \mathrm{C}$, and the white homogenous polymer in the powder form was received. Soxhlet extraction was then performed to elute the template molecules (i.e., TBZ) from the cavities of the polymer. Specifically, $200 \mathrm{~mL}$ methacrylic acid/methanol $(5: 5, \mathrm{v} / \mathrm{v})$ was used for Soxhlet extraction during the first 24 hours, followed by another $200 \mathrm{~mL}$ methanol to completely remove template molecules and wash off acetic acids for another 24 hours. The resultant MIPs were dried in a vacuum oven at $50^{\circ} \mathrm{C}$ for 12 hours. The non-imprinted polymers (NIPs) were also produced following the same procedures without the addition of template molecules (i.e., TBZ).

\subsubsection{Characterization of MIPs}

The static adsorption capacity test was conducted as follows. Ten milligrams of MIPs or NIPs were mixed with $2 \mathrm{~mL}$ of each TBZ standard solution $(0,10,25,50$ and $75 \mathrm{ppm}$ in $20 \%$ acetonitrile). The maximum UV absorption $(\lambda=298.5 \mathrm{~nm})$ was determined by scanning the standard solution from $200 \mathrm{~nm}$ to $800 \mathrm{~nm}$ in a Shimadzu UV-Vis spectrophotometer (Shimadzu corporation, Kyoto, Japan). The mixture was shaken at $200 \mathrm{rpm}$ for 12 hours at room temperature, followed by centrifugation at $12,000 \times g$ for 5 minutes. The absorption of the 
supernatant was determined at a wavelength of $298.5 \mathrm{~nm}$. Two batches of the synthesized MIPs and NIPs were individually tested in triplicate to evaluate the rebinding capacity.

The kinetic adsorption capacity test was performed by mixing $10 \mathrm{mg}$ of MIPs or NIPs with 10 ppm TBZ solution $(2 \mathrm{~mL})$ and then shaken at $200 \mathrm{rpm}$ for different time intervals $(5,15,30,60$, $90,120,180$ and $240 \mathrm{~min})$. The mixture $(1 \mathrm{~mL})$ was centrifuged at $12,000 \times g$ for $5 \mathrm{~min}$, and the absorption of the supernatant was determined at $298.5 \mathrm{~nm}$ using the UV-Vis spectrophotometer.

\subsubsection{Food sample preparation}

Sample preparation was based upon the salting-out technique, as indicated in a previous work ${ }^{35}$. Orange juice $(10 \mathrm{~mL})$ spiked with $\operatorname{TBZ}(0,10,25,50$, and $75 \mathrm{ppm})$ was mixed with $1 \%$ acetic acid in acetonitrile $(10 \mathrm{~mL})$. Magnesium sulfate $(4 \mathrm{~g})$ and sodium acetate $(1.7 \mathrm{~g})$ were then added to the mixture, followed by centrifugation at $2040 \times g$ for $8 \mathrm{~min}$. The supernatant was collected for further analysis.

\subsubsection{Molecularly imprinted solid phase extraction (MISPE)}

MIPs or NIPs powder $(150 \mathrm{mg}$ ) were packed into a $3 \mathrm{~mL}$ polypropylene SPE cartridges with a PTFE frit on the top and a membrane $(0.45 \mu \mathrm{m})$ at the bottom. The SPE cartridge was conditioned with $2 \mathrm{~mL}$ water and then $2 \mathrm{~mL}$ pure methanol. Each sample ( $2 \mathrm{~mL})$ was loaded, followed by addition of $3 \mathrm{~mL} \mathrm{50 \%} \mathrm{methanol} \mathrm{to} \mathrm{wash} \mathrm{off} \mathrm{inferences} \mathrm{from} \mathrm{the} \mathrm{column.} \mathrm{Finally,}$ TBZ was eluted out by $3 \mathrm{~mL}$ pure methanol. The flow rate was controlled at $1 \mathrm{~min} \mathrm{~L}^{-1}$ for each step by the application of vacuum to SPE manifold. An aliquot of eluent $(0.24 \mu \mathrm{L})$ was mixed 
with an aliquot of homemade silver colloid $(0.96 \mu \mathrm{L})$, and then directly deposited onto a goldcoated microarray chip for Raman spectral collection.

\subsubsection{High-performance liquid chromatography}

HPLC analysis was conducted using an Agilent 1100 series HPLC system that consists of a quaternary pump and a diode array detector set at $280 \mathrm{~nm}$. Eluent solution $(5 \mu \mathrm{L})$ was injected into a reverse phase column (Kinetex® $2.6 \mu \mathrm{m} \mathrm{C}_{18} 100 \AA$, LC Column 100 x $3 \mathrm{~mm}$ ) at $25^{\circ} \mathrm{C}$. The mobile phase was ultrapure water-methanol $(35: 65, \mathrm{v} / \mathrm{v})$ with the flow rate set at $0.2 \mathrm{~mL}$ $\min ^{-1}$. Running time for each sample was $8 \mathrm{~min}$. Each sample was tested at least in triplicate.

\subsubsection{Synthesis of silver colloid}

The synthesis of silver colloid as SERS substrate was followed by a procedure described in a previous work ${ }^{30}$ with modification. $\mathrm{AgNO}_{3}(54 \mathrm{mg})$ was dissolved in $300 \mathrm{~mL} \mathrm{H}_{2} \mathrm{O}$ in a round bottom flask. Reflux system was set up with an oil bath and nitrogen bubbling. Then, $1 \%$ trisodium citrate $(30 \mathrm{~mL})$ was added to the solution at $90^{\circ} \mathrm{C}$. The mixture was refluxed and stirred at $120^{\circ} \mathrm{C}$ for $90 \mathrm{~min}$. When the color of the solution turned to turbid gray-green, the mixture was removed from heat and cooled down to room temperature with stirring.

\subsubsection{Scanning electron microscopy (SEM)}

SEM was conducted to characterize the morphology of the synthesized MIPs, NIPs, and silver colloid, respectively, at University of British Columbia Bioimaging Facility. During sample preparation for SEM characterization, some nano-sized Palladium (Pd) powers were sprayed onto MIPs and NIPs to induce electric conductivity. For Ag colloid, no coating was applied. 


\subsubsection{Raman spectral collection and multivariate statistical analysis}

Raman spectral collection in a wavenumber region of 400 to $2000 \mathrm{~cm}^{-1}$ was conducted using a 785-nm diode laser (25-mW incident laser power) coupled with a 50× objective. The Raman spectrometer (Renishaw, Gloucestershire, UK) has an entrance aperture of $50 \mu \mathrm{m}$, a focal length of $300 \mathrm{~mm}$, and is equipped with a 1200 -line $\mathrm{mm}^{-1}$ grating. A 578- by 385-pixel charge coupled device (CCD) array detector with a pixel size of $22 \mu \mathrm{m}$ was provided to collect Raman signal. A total of 15 spectra per sample were collected to study spectral reproducibility as well as construct chemometric models.

OMNIC software version 7.0 (Thermo Electron Corp., Madison, U.S.A.) was used for spectral processing and analysis. To reduce the spectral noise, automatic baseline correction and spectral smoothing (11-point Savitzky-Golay algorithm) were applied for raw Raman spectra. Unsupervised principal component analysis (PCA) was used to differentiate non-spiked orange juice sample from orange juice samples containing different concentrations of TBZ. In addition, partial least squares regression (PLSR) model was established and validated via "leave-one-out" cross-validation method to quantify the actual content of TBZ in orange juice. MATLAB v. R2016a (The MathWorks, Inc., USA) was used to develop both PCA and PLSR models.

\subsection{Results and discussion}

\subsubsection{Synthesis of TBZ-imprinted polymers}

Based upon a previous study with further modification ${ }^{36}$, TBZ-imprinted polymers was synthesized by using precipitation polymerization method. MAA was selected as the functional 
monomer to interact with a basic template molecule (i.e., TBZ) that prefers to form a prepolymerization complex in the solvent. The selected solvent was acetonitrile: toluene $(75: 25$, $\mathrm{v} / \mathrm{v})$, and it also worked as the porogen to facilitate the formation of spatial pores in the developed polymer. According to the previous work, toluene can affect the porosity in MIPs that leads to a higher surface area. Matching solubility parameter of toluene to that of the crosslinking agent is also vital to the formation of the regular shape of MIPs ${ }^{37}$. Hence, DVB was used as the crosslinker because it has similar solubility to toluene. Besides, the half-life time of AIBN at $60^{\circ} \mathrm{C}$ was $\sim 10 \mathrm{~h}$, which is appropriate as the polymerization time ${ }^{38}$. Thus, it was used as the initiator for thermal polymerization. The synthesis process of MIPs is shown in Figure 3.1. In TBZ molecule, two N-atoms (hydrogen acceptor) can form H-bond with a hydroxyl group in MAA, and N-H group (hydrogen donor) can form H-bond with the carbonyl group in MAA. Without strong intra-molecular hydrogen bonding, all of these three sites have intermolecular $\mathrm{H}$ bonds with the functional monomer to form precise imprinting sites. After the removal of the template, the binding sites are left with specific recognition to the template molecule. 

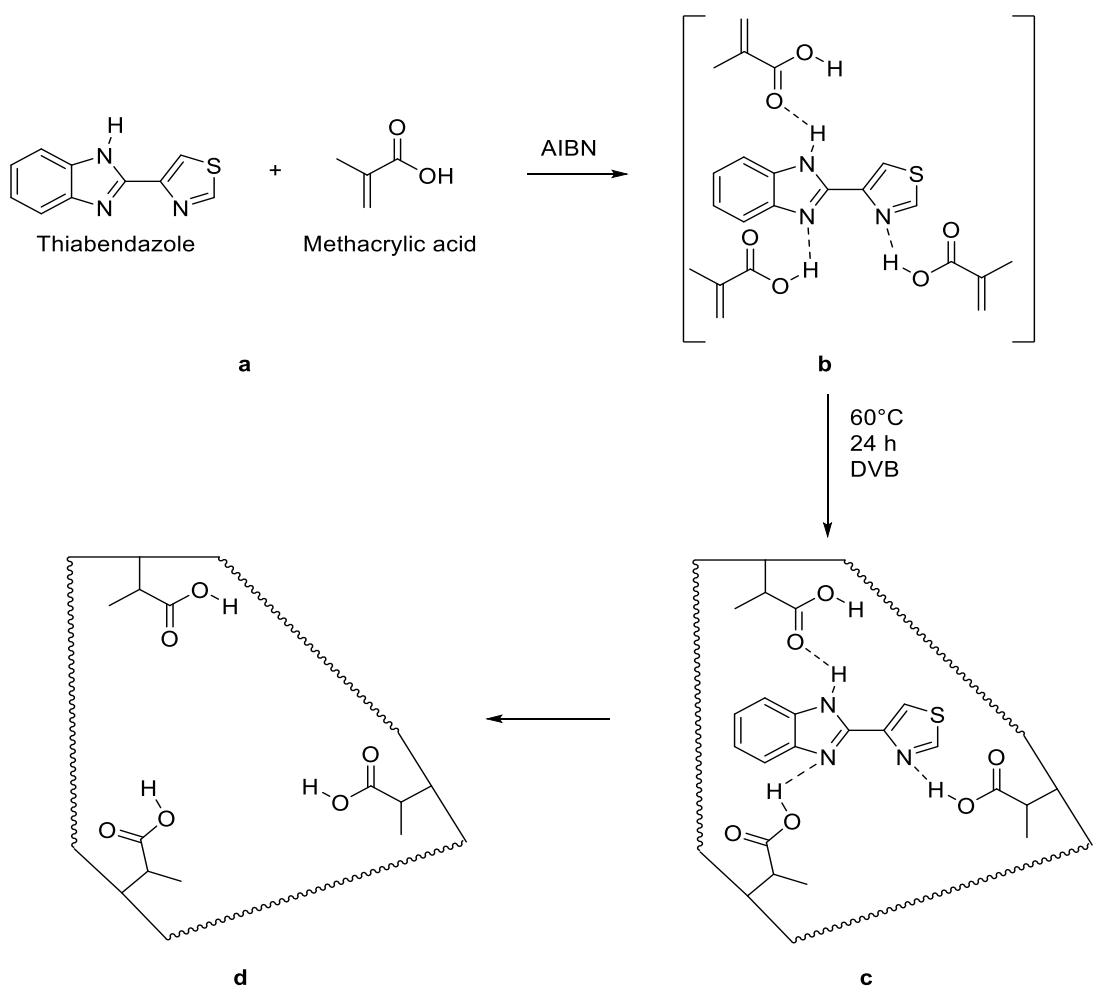

Figure 3.1 The synthesis process of MIPs towards TBZ. (a) Starting materials are listed as follow: template molecule (thiabendazole), functional monomer (methacrylic acid), and initiator (AIBN: 2, 2azobisisobutyronitrile). (b) Proposed hydrogen bonding will form between a template molecule and functional monomers in the pre-polymerization stage. (c) Polymerization with cross-linking agents is carried out in the presence of the complex. (d) Subsequent removal of the template yields a binding site complementary to the template molecule.

\subsubsection{Binding affinity of TBZ-imprinted polymers}

The adsorption tests were conducted to investigate the binding performance of TBZ-imprinted polymers. The selection of solvent was carried out to optimize the solvent system before adsorption test. A good solvent system with moderate solubility to the analyte can aid in the dispersion of MIPs in the solution and facilitate the interaction between analytes and MIPs. Acetonitrile is a polar aprotic solvent and shows good solubility to TBZ, resulting in a decrease 
in the non-specific interaction (e.g., hydrophobic interaction). In our preliminary study, $10 \mathrm{mg}$ of MIPs or NIPs were mixed with $10 \mathrm{ppm}$ TBZ in the aqueous solution with different percentages of acetonitrile. The adsorption capacities of both MIPs and NIPs towards TBZ were determined. Compared to MIPs, less TBZ rebound to NIP due to non-specific binding solely. Hence, the absorbance of supernatant in NIPs is higher than that in MIPs. The ratio of absorbance of supernatant in NIPs to that in MIPs is shown in Figure 3.2. The greater of the ratio, the larger difference was between MIPs and NIPs. The ratio of $20 \%$ acetonitrile was the highest (i.e., 1.45). Therefore, $20 \%$ acetonitrile was selected as the solvent to study the adsorption capacity of MIPs.

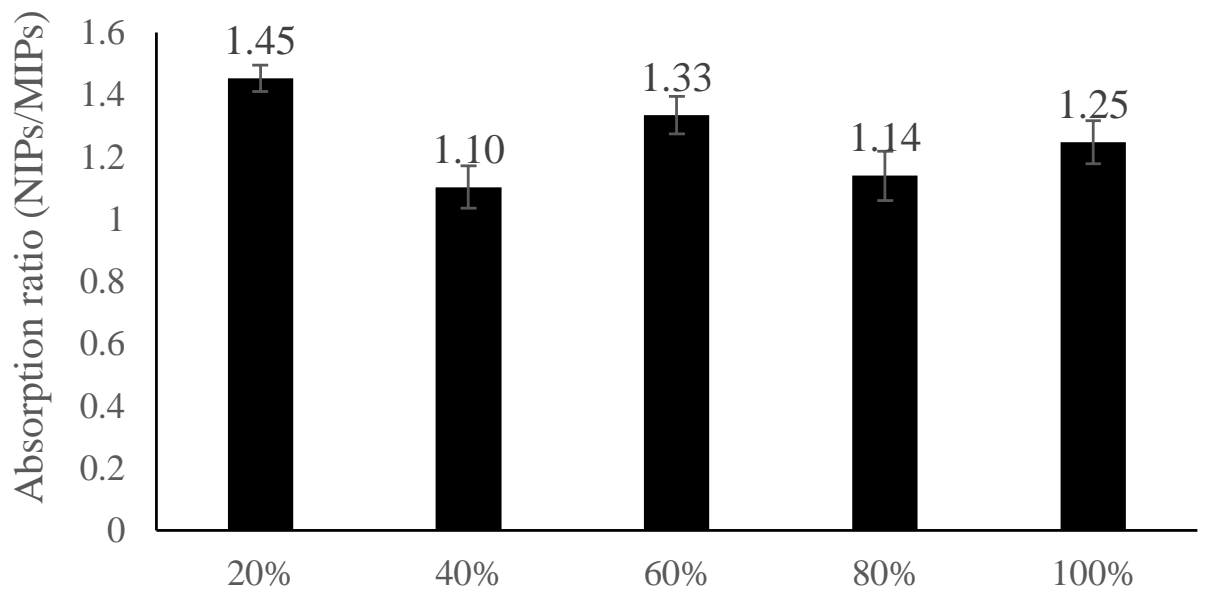

Acetonitrile percentage in aqueous solution

Figure 3.2 The ratio of absorbance of supernatant in NIPs to that in MIPs towards 10 ppm TBZ after 3-h incubation as a function of the percentage of acetonitrile in the aqueous solution.

Adsorption capacity $(\mathrm{Q})$ is the primary parameter to evaluate the binding performance of the developed polymers. The following equation can be carried out to calculate the adsorption capacity towards TBZ: 


$$
\mathrm{Q}=\left[\left(\mathrm{C}_{\mathrm{i}}-\mathrm{C}_{\mathrm{f}}\right) \times \mathrm{V} / \mathrm{W}\right]
$$

Equation 3.1

, where $\mathrm{C}_{\mathrm{i}}$ and $\mathrm{C}_{\mathrm{f}}\left(\mathrm{ppm}\right.$ or $\left.\mathrm{mg} \mathrm{L}^{-1}\right)$ are the initial and final concentrations of $\mathrm{TBZ}$ in the solution, respectively, $\mathrm{Q}\left(\mathrm{mg} \mathrm{g}^{-1}\right)$ is the adsorption capacity, $\mathrm{V}(\mathrm{mL})$ is the volume of the solution, and $\mathrm{W}$ is the mass of the polymer ${ }^{39}$.

Regardless of the initial concentration of TBZ, MIPs yielded higher adsorption capacity values than NIPs. The difference of adsorption capacity between MIPs and NIPs increased from 0.19 $\mathrm{mg} \mathrm{g}^{-1}$ to $0.30 \mathrm{mg} \mathrm{g}^{-1}$ along with the increase in the concentration of TBZ in Figure $3.3 \mathrm{a}$. The relationship between adsorption capacity and initial concentration of TBZ was linear for MIPs, indicating that the developed cavities and specific binding in MIPs were stable.

Scatchard plot is commonly used to access the affinity of polymers. The Scatchard equation can be expressed as follows:

$$
\mathrm{Q} / \mathrm{C}_{\mathrm{i}}=-\mathrm{Q} / \mathrm{K}_{\mathrm{d}}+\mathrm{Q}_{\max } / \mathrm{K}_{\mathrm{d}}
$$

Equation 3.2

, where $\mathrm{Q}_{\max }\left(\mathrm{mg} \mathrm{g}^{-1}\right)$ is the maximum amount of adsorption and $\mathrm{K}_{\mathrm{d}}\left(\mathrm{mg} \mathrm{L}^{-1}\right)$ is the dissociation equilibrium constant at the imprinted sites ${ }^{38}$.

The Scatchard plot of MIPs and NIPs are shown in Figure 3.3 b. The relationship of Q/C vs. Q was linear for MIPs $\left(\mathrm{R}^{2}=0.93\right)$. The maximum adsorption capacity $\left(\mathrm{Q}_{\max }\right)$ calculated from this plot was $6.31 \mathrm{mg} \mathrm{g}^{-1}$. This linear relationship was due to the hydrogen bonds formed between 
specific binding sites in MIPs and the analyte molecules (i.e., TBZ). For NIPs, there was no direct correlation because the adsorption capacity of NIPs was derived from non-specific binding, such as hydrophobic interaction.

Kinetic adsorption test was also performed to assess the rate of the development of specific bonds between the analyte molecule (i.e., TBZ) and MIPs. The adsorption process could reach to equilibrium within 5 min (Figure $3.3 \mathrm{c}$ ), indicating that the special bonds can be formed rapidly and MIPs are ideal for rapid and efficient separation of the targeted analytes. 

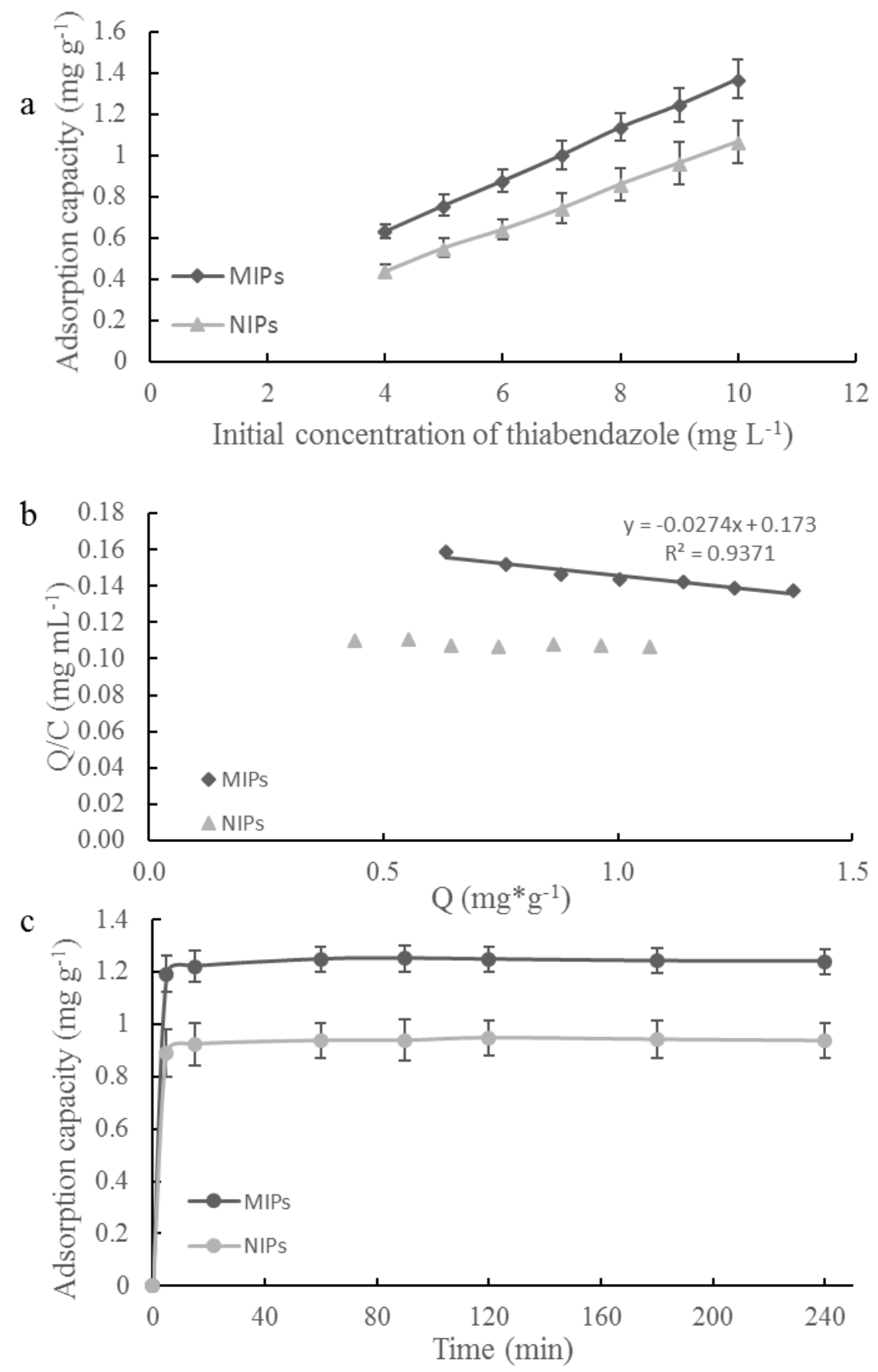

Figure 3.3 (a) Static binding isotherm of molecularly imprinted polymers (MIPs) and non-imprinted polymers (NIPs) towards thiabendazole; (b) Scatchard plot of MIPs and NIPs toward thiabendazole; (c) Kinetic binding isotherm of MIPs and NIPs (Ci: $\left.10 \mathrm{mg} \mathrm{L}^{-1}\right)$. Ci: initial concentrations of thiabendazole; Q: binding capacity. Error bars indicate standard deviation $(\mathbf{N}=\mathbf{3})$. 


\subsubsection{Performance of MISPE}

There are many components existed in orange juice, such as sugars, dietary fibers in pulps, vitamins, and minerals (e.g., $\mathrm{Ca}, \mathrm{Mn}, \mathrm{Fe}, \mathrm{P}, \mathrm{K}, \mathrm{Na}$, and $\mathrm{Zn}$ ). Therefore, a sample pre-treatment step was performed to remove the macromolecules and pulps through the salting-out liquid and liquid partition. As shown in Figure 3.4, different separated layers could develop after sample pre-treatment. Specifically, the supernatant was the acetonitrile layer with the extracted analyte (i.e., TBZ); the second layer was the homogenized pulps while the third layer was the aqueous layer with saturated buffer salts and ionic/polar matrices. The bottom layer was the undissolved buffer salts layer. Hence, the supernatant was transferred into a clean vial for further use. This sample preparation procedure only took $10 \mathrm{~min}$ in total.

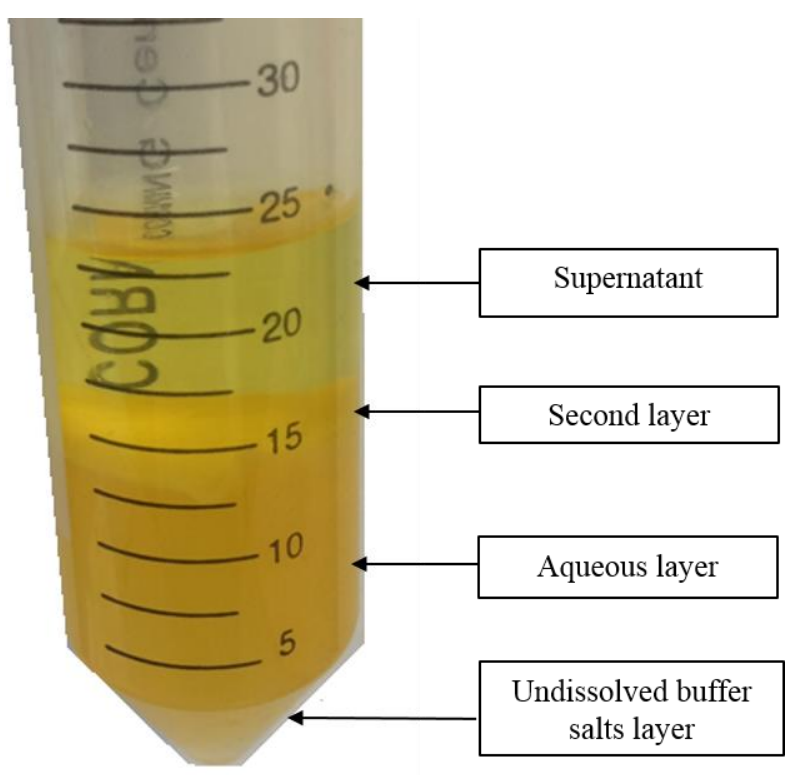

Figure 3.4 The separation layers of orange juice after sample preparation.

The principle of MISPE for the extraction of TBZ from orange juice is shown in Figure 3.5. The synthesized MIPs powders were packed into an SPE cartridge. After conditioning with water and methanol, the supernatant $(2 \mathrm{~mL})$ was loaded onto the column at a flow rate of $1 \mathrm{~mL} \mathrm{~min}{ }^{-1}$. This 
flow rate can provide a sufficient interaction between analyte and MIPs. Pure methanol and water were then applied to remove the polar matrices. The elution was subsequently performed to break the hydrogen binding between TBZ and MIPs, and the eluent was collected for further analysis. The whole process took 12 min, which is much faster than a mixed-mode SPE for the separation of TBZ, as described in a previous study $(\sim 2 \mathrm{~h})^{25}$.

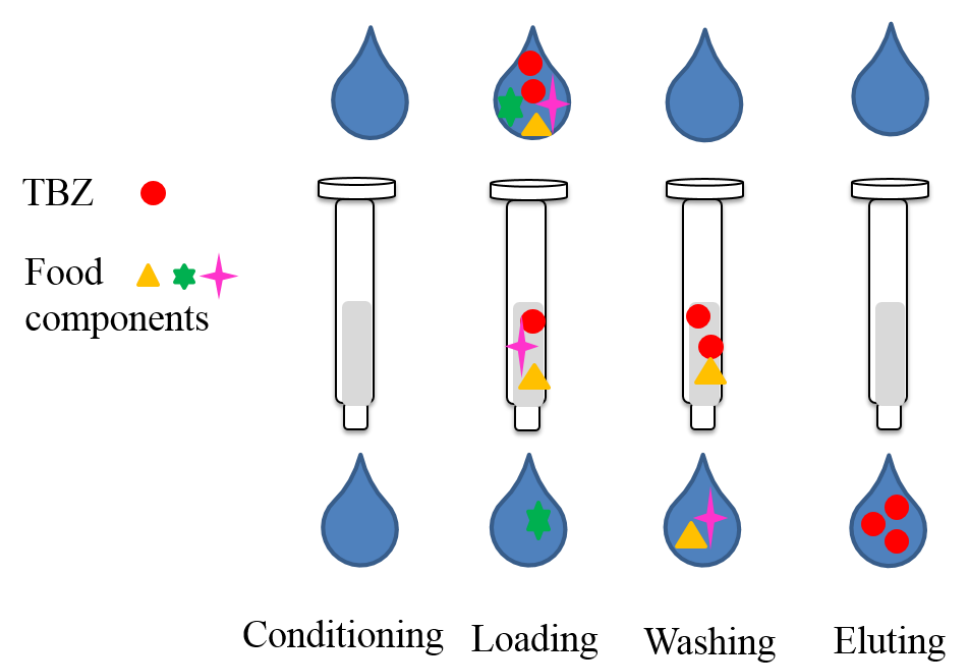

Figure 3.5 Schematic illustration of molecularly imprinted solid phase extraction (MISPE) of TBZ in orange juice.

Recovery test was conducted to validate the efficiency of MISPE column. The average recovery of TBZ in orange juice after MISPE or NISPE column was determined by using HPLC-DAD. The result of recovery test is summarized in Table 3.1. The recovery of MISPE was over $87 \%$ for TBZ with a good reproducibility. At a lower concentration of TBZ, the recovery was relatively higher because the saturation of binding capacity was achieved in a relatively high concentration of TBZ. The difference in recovery between MISPE and NISPE was between 40$55 \%$ at a flow rate of $1 \mathrm{~mL} \mathrm{m^{-1 }}$, validating the specific binding between MISPE column and TBZ. 
Table 3.1. The average recovery of thiabendazole (TBZ) in orange juice by molecularly imprinted solid phase extraction (MISPE) and non-imprinted solid phase extraction (NISPE) (N=3).

$\begin{array}{ccc}\text { Concentration }\left(\mathrm{mg} \mathrm{L}^{-1}\right) & \text { Recovery of MISPE }(\%) & \text { Recovery of NISPE (\%) } \\ 0 & \text { N/A } & 50.21 \\ 10 & 97.09 & 53.79 \\ 25 & 108.81 & 46.89 \\ 50 & 89.44 & 46.47\end{array}$

\subsubsection{Quantitative analysis of TBZ in orange juice by SERS}

Silver colloids were synthesized via chemical reduction of silver nitrate with citrate as the reducing agent. A mixture of a quasi-spherical and small amount of rod-like nanoparticles can be observed by using scanning electron microscopy (Figure 3.6). With over 30 measurements, the size distribution of Ag nanoparticles was calculated to be $41.4 \pm 9.1 \mathrm{~nm}$ via Image $\mathrm{J}$. The size and shape distribution was due to the fast reduction of $\mathrm{Ag}^{+}$precursor under a relatively high $\mathrm{pH}$ value (i.e., 7.7) ${ }^{40}$. The synthesized Ag colloids were then used as the SERS-active substrate to induce a significant enhancement of Raman scattering signals from the samples containing TBZ 30. 


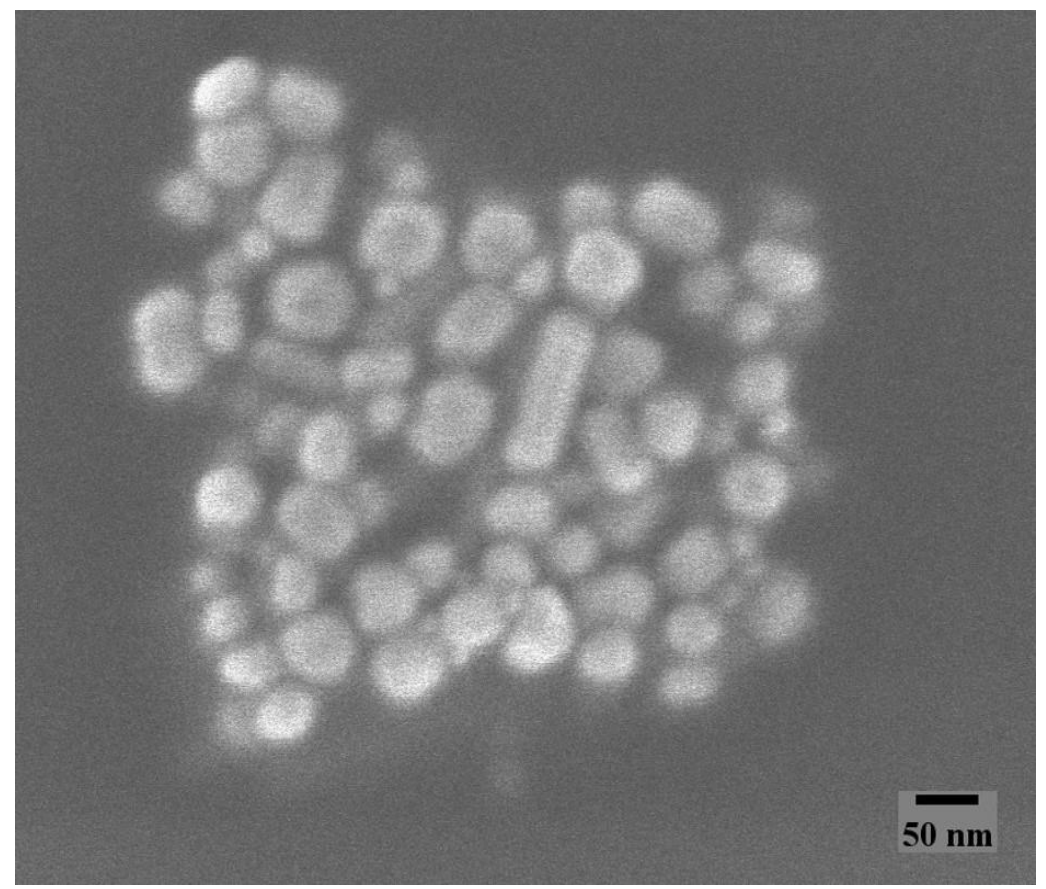

Figure 3.6 Representative scanning electron microscope image for sliver collide.

The mixture of Ag colloid $(0.24 \mu \mathrm{l})$ and eluted orange juice sample $(0.96 \mu \mathrm{l})$ was deposited onto a gold-coated microarray chip, followed by SERS spectral collection. The average normal Raman spectrum (N=15) of pure TBZ power is shown in Figure 3.7. The most intense peak was observed at $1573 \mathrm{~cm}^{-1}$ and it was derived from multiple vibration modes, such as $\mathrm{C}-\mathrm{C}$ stretching in entire molecule, $\mathrm{N}=\mathrm{C}, \mathrm{C}=\mathrm{C}$ stretching, and $\mathrm{C}-\mathrm{H}, \mathrm{C}-\mathrm{N}-\mathrm{H}$ bending in ring $2, \mathrm{C}-\mathrm{H}$ bending in ring 3 , and $\mathrm{N}-\mathrm{C}$ bending in ring $1^{41}$. The peak at $783 \mathrm{~cm}^{-1}$ was derived from $\mathrm{S}-\mathrm{C}$ stretching mode and $\mathrm{C}=\mathrm{N}$ bending mode of ring $1^{41}$. The characteristic peak at $1281 \mathrm{~cm}^{-1}$ was assigned to $\mathrm{C}-\mathrm{N}$ stretch for ring $2, \mathrm{C}-\mathrm{C}$ stretch for ring 1 , and bending mode of ring $2^{41}$.

As shown in Figure 3.7, SERS spectral feature for standard TBZ methanol solution $\left(50 \mathrm{mg} \mathrm{L}^{-1}\right)$ was in consistent to the feature of normal Raman spectra of TBZ power. The enhance factor was 
calculated to be $\sim 10^{4}$ compared to the normal Raman spectra. In theory, the enhancement comes from sharing $\pi$ electron from rings and sharing lone pair electron on $\mathrm{N}$ and $\mathrm{S}$ atom between TBZ and the surface of Ag nanoparticles ${ }^{41}$. Hence, we propose that TBZ attaches itself onto the surface of silver colloids in two ways, namely standing up orientation and planar coverage of surface (Figure 3.8).

The SERS spectrum of eluent orange juice spiked with $50 \mathrm{mg} \mathrm{L}^{-1} \mathrm{TBZ}$ form MISPE is also shown in Figure 3.7. Compared to the normal Raman spectral counterpart, citrate peak originated from the citrate-reduced silver colloid was present in both SERS and MISPE-SERS spectra at $1050 \mathrm{~cm}^{-1}$ that correspond to $\mathrm{C}-\mathrm{O}$ vibration ${ }^{42}$. In both SERS spectra, peaks at $1281 \mathrm{~cm}^{-1}$ and $1573 \mathrm{~cm}^{-1}$ were broadened, which was due to the parallel orientation of molecule skeleton to the surface of silver nanoparticles. Compared to the standard SERS spectrum, the peaks in the MISPE-SERS spectrum were consistent, validating the efficiency of performing MISPE to remove most of the components in orange juice. The minor Raman peaks derived from the orange juice residues can be assigned to the wavenumber region between $1350 \mathrm{~cm}^{-1}$ and 1450 $\mathrm{cm}^{-1}$. 


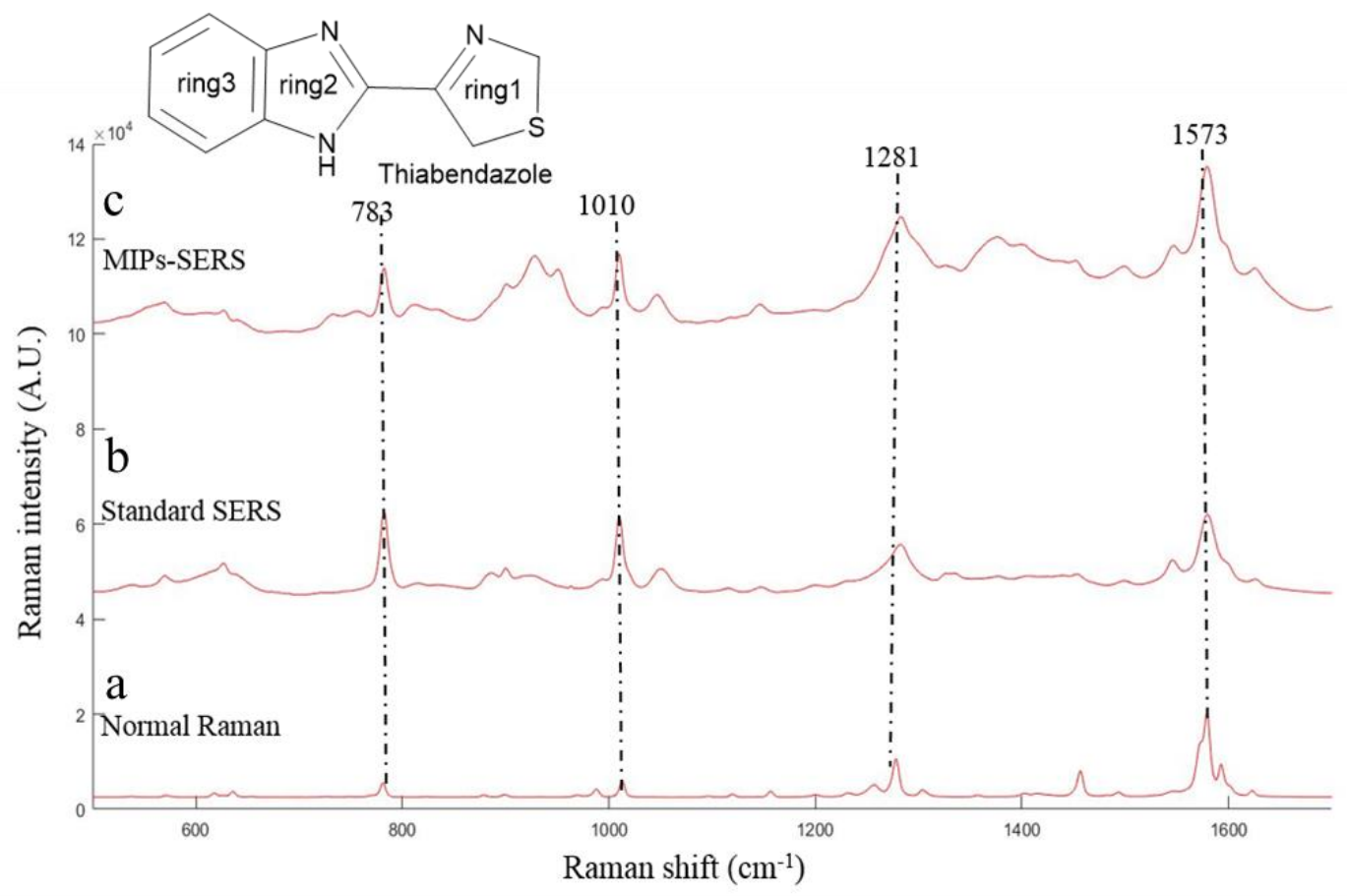

Figure 3.7 Representative spectral features of thiabendazole (TBZ) with (a) normal Raman of TBZ powder, (b) SERS of standard TBZ solution in methanol (50 $\left.\mathrm{mg} \mathrm{L}^{-1}\right)$, and (c) MISPE-SERS of orange juice spiked with $50 \mathrm{mg} \mathrm{L}^{-1} \mathrm{TBZ}$.

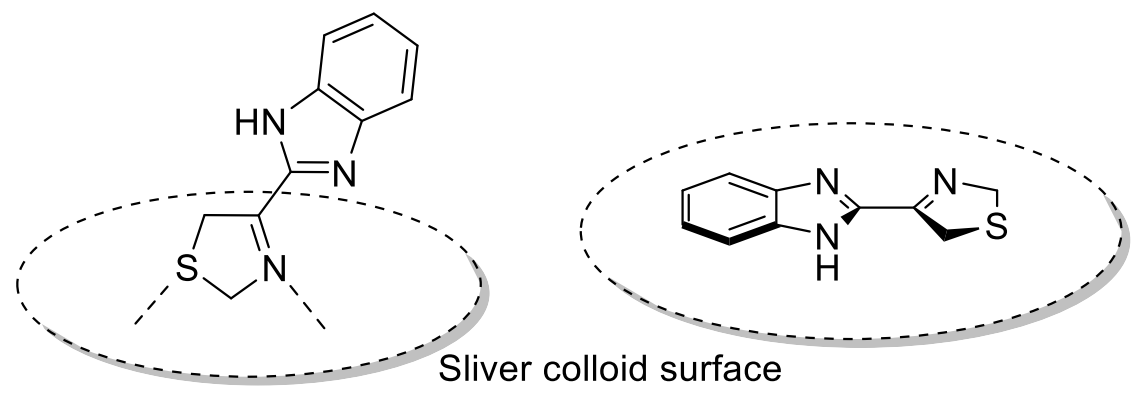

Figure 3.8 Schematic illustration of the orientation model of TBZ onto the surface of silver colloid. Left: The lone pair electrons on $\mathrm{S}$ and $\mathrm{N}$ atom result in a standing-up orientation of $\mathrm{TBZ}$ to the surface of $\mathrm{Ag}$ nanoparticles. Right: The $\pi$ ring electrons result in a planar coverage of the surface by TBZ.

MIPs-SERS spectra for orange juice spiked with different concentrations of TBZ were preprocessed via smoothing and automatic baseline correction. The average SERS spectra 
$(\mathrm{N}=15)$ are shown in Figure 3.9. The intensity of the characteristic peaks was increased along with the increase in the concentration of TBZ spiked in juice. For orange juice without the spiked TBZ (0 ppm), no major peaks related to the analyte appeared in the spectra.

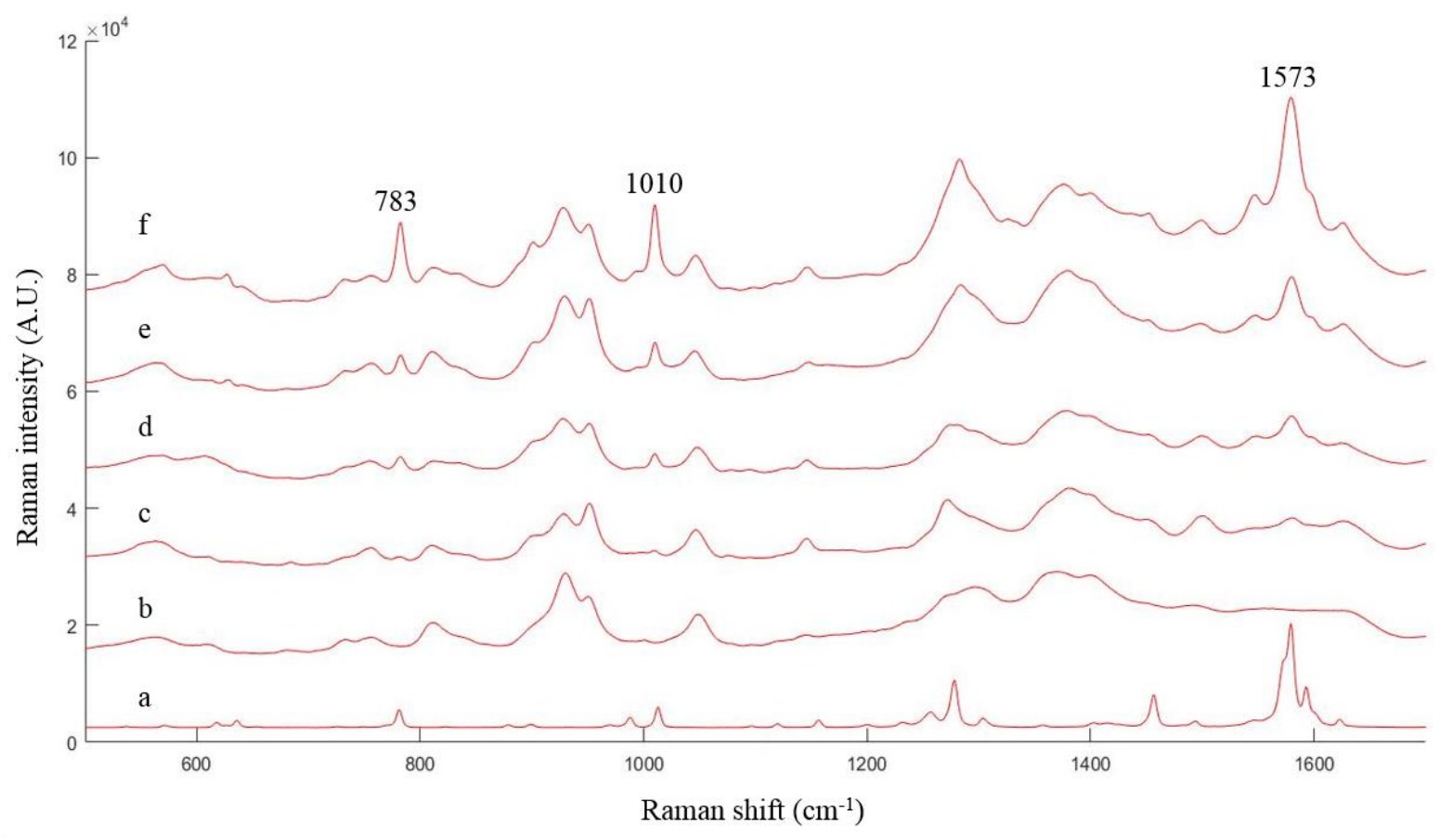

Figure 3.9 Representative normal Raman spectral features with (a) thiabendazole powder, Representative MIPs-SERS spectra of orange juice spiked with different concentrations of thiabendazole: (b) $0 \mathrm{mg} \mathrm{L}^{-1}$, (c) 10 $\mathrm{mg} \mathrm{L} \mathbf{L}^{-1}$, (d) $25 \mathrm{mg} \mathrm{L}^{-1}$, (e) $50 \mathrm{mg} \mathrm{L}^{-1}$, and (f) $75 \mathrm{mg} \mathrm{L}^{-1}$.

Unsupervised two-dimensional principal component analysis (PCA) model was established to differentiate the non-spiked orange juice sample $(0 \mathrm{ppm})$ from the orange juice samples that were spiked with different concentrations of $\operatorname{TBZ}(10,25,50$, and $75 \mathrm{ppm})$. The representative PCA model is shown in Figure 3.10. For each spiked concentration, 15 spectra were collected, followed by spectral processing and baseline correction. Orange juice samples spiked with each 
concentration of TBZ were tightly clustered and well separated from each other, indicating that PCA model can be applied to differentiate the spiking level of TBZ in orange juice.

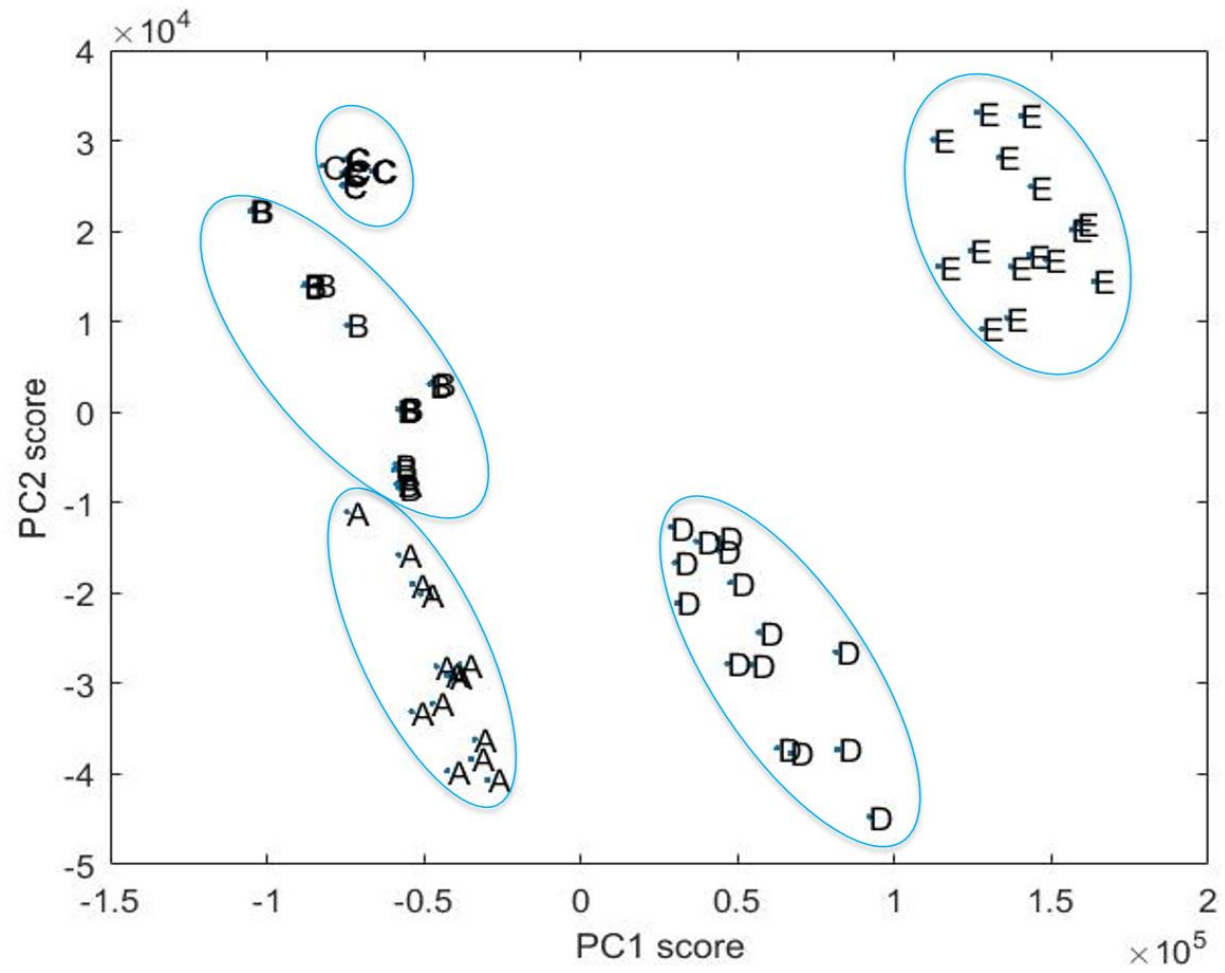

Figure 3.10 Two-dimensional principal component analysis for orange juice spiked with different concentrations of thiabendazole (TBZ). Principal component (PC)1 represents 86.3\% variances and PC2 represents $7 \%$ variances. Letters from A to $\mathrm{E}$ denote orange juice spiked with $\mathrm{TBZ}$ at the concentration of 0 , $10,25,50$ and $75 \mathrm{mg} \mathrm{L}^{-1}$, respectively.

Partial least squares regression (PLSR) model was then applied to quantify the spiked level of TBZ in orange juice (Figure 3.11). The linear relationship $\left(\mathrm{R}^{2}=0.99\right)$ validated the accurate prediction of TBZ concentration in orange juice by using this PLSR model. The LOD was calculated to be 4 ppm, which was lower than the MRL value (i.e., $10 \mathrm{ppm}$ ). Taken together, this MISPE-SERS chemosensor could be applicable for the determination of trace amount of TBZ in orange juice. 
Table 3.2. Partial least squares regression (PLSR) models for the prediction of thiabendazole concentration in orange juice.

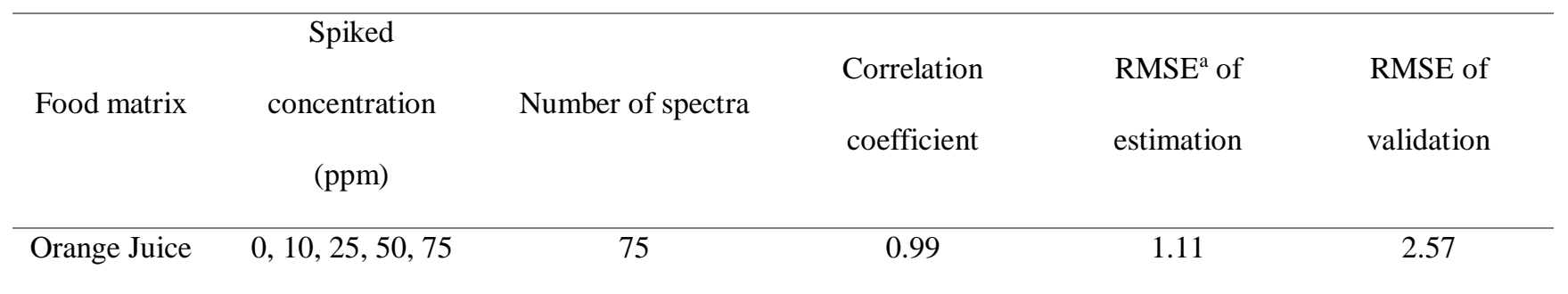

a. RMSE: root mean square error.

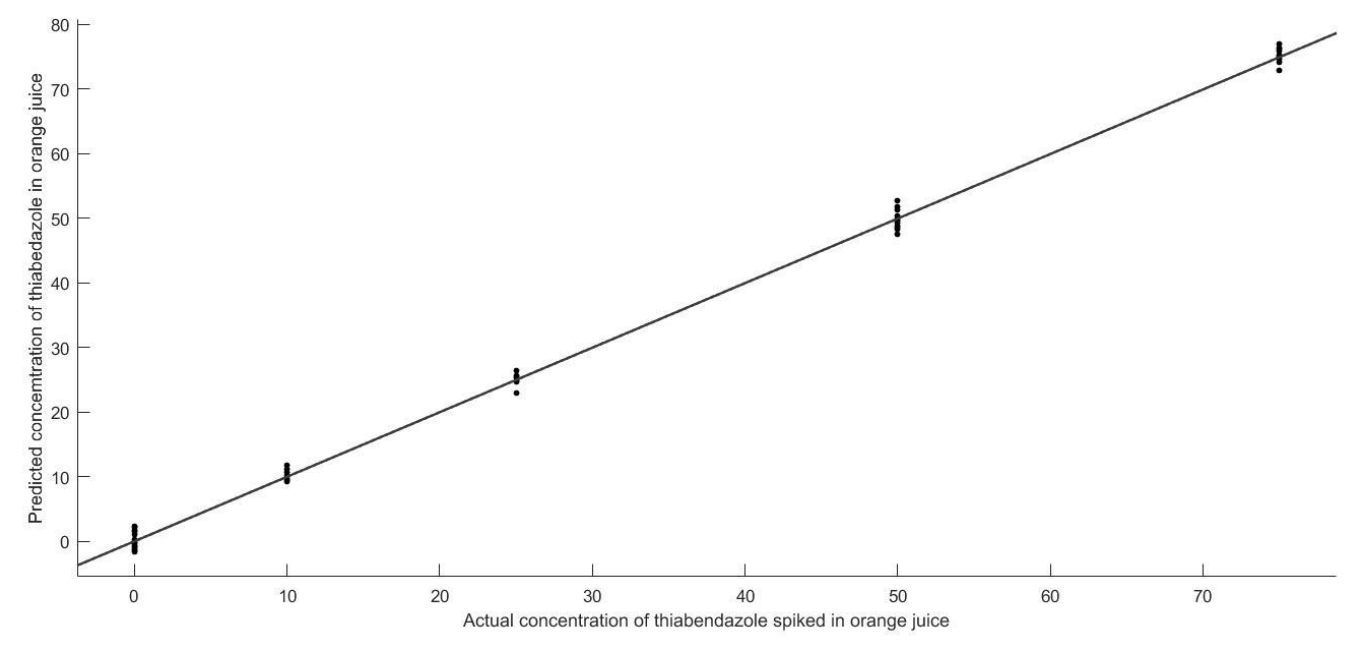

Figure 3.11 Partial least squares regression (PLSR) model predicts the actual concentrations of TBZ in orange juice.

In this work, 10 min was applied for sample preparation, followed by another 12 min for MISPE to separate TBZ from orange juice. SERS required $<1$ min per spectral collection. The whole process by applying MISPE-SERS chemosensor to detect TBZ in orange juice could be completed within 23 min, including sample preparation. In comparison, SPE coupled with the 
conventional detection method requires several hours due to the complicated sample preparation along with an extensive time for detection.

\subsection{Conclusion}

In this thesis project, we for the first time fabricated an innovative MISPE-SERS chemosensor for rapid, accurate, and sensitive determination of TBZ in orange juice. The whole process of this MISPE-SERS chemosensor only took 23 min with the LOD of $4 \mathrm{ppm}$ for TBZ. In sum, this MISPE-SERS chemosensor can be considered as a promising method for fast and highthroughput detection of trace amount of chemical hazards in agri-food products. 


\section{Bibliography}

(1) Yang, T.; Zhang, Z.; Zhao, B.; Hou, R.-Y.; Kinchla, A.; Clark, J. M.; He, L. Real-time and in situ monitoring of pesticide penetration in edible leaves by surface-enhanced Raman scattering mapping. Anal. Chem. 2016, acs.analchem.6b00320.

(2) Health Canada. Proposed Re-evaluation Decision PRVD2010-12 Thiabendazole; 2010.

(3) Polyakov, M. V. Adsorption properties and structure of silica gel. Zhurnal Fizieskoj Khimii/Akademiya SSSR 1931, 2, 799-805.

(4) Wulff, G.; Sarhan, A. The use of polymers with enzyme-analogous structures for the resolution of racemates. Angew. Chemie Int. Ed. 1972, 11 (4), 341.

(5) Shea, K. J.; Thompson, E. A. Template synthesis of macromolecules. Selective functionalization of an organic polymer. J. Org. Chem. 1978, 43 (21), 4253-4255.

(6) Shea, K.; Thompson, E. Template synthesis of macromolecules. Synthesis and chemistry of functionalized macroporous poly (divinylbenzene). Am. Chem. Soc. 1980, 102 (2), $3148-3156$.

(7) Damen, J.; Neckers, D. C. Memory of synthesized vinyl polymers for their origins. J. Org. Chem. 1980, 45 (8), 1382-1387.

(8) Reza, A.; Klaus, M. Synthesis of substrate-selective polymers by host-guest polymerization. Macromol. Chem. Phys. 1981, 182 (2), 687-692.

(9) Vasapollo, G.; Sole, R. Del; Mergola, L.; Lazzoi, M. R.; Scardino, A.; Scorrano, S.; Mele, G. Molecularly imprinted polymers: Present and future prospective. Int. J. Mol. Sci. 2011, 12 (9), 5908-5945.

(10) Mayes, A. G.; Whitcombe, M. J. Synthetic strategies for the generation of molecularly imprinted organic polymers. Adv. Drug Deliv. Rev. 2005, 57 (12), 1742-1778. 
(11) Magnetism, R.; Dunlop, D. J. Chapter 2 Fundamentals of magnetism. In Molecular Imprinting: From Fundamentals to Applications; 2004; Vol. 1, pp 16-44.

(12) Komiyama, M.; Takeuchi, T.; Mukawa, T.; Asanuma, H. Chapter 3 Experimental Methods (1) - Procedures of Molecular Imprinting. In Molecular Imprinting: From Fundamentals to Applications; 2004; pp 21-45.

(13) Mulawka, M. Chapter 1 - Introduction. In Wiley Online Library You have full-text access to this content Molecular Imprinting: From Fundamentals to Applications; 2004; pp 1-8.

(14) Ye, L.; Haupt, K. Molecularly imprinted polymers as antibody and receptor mimics for assays, sensors and drug discovery. Anal. Bioanal. Chem. 2004, 378 (8), 1887-1897.

(15) Lofgreen, J. E. Controlling morphology and porosity to improve performance of molecularly imprinted sol-gel silica. Chem. Soc. Rev. 2014, 43 (3), 911-933.

(16) John William Strutt, 3rd Baron Rayleigh https://en.wikipedia.org/wiki/John_William_Strutt,_3rd_Baron_Rayleigh.

(17) Vandenabeele, P. 1.1 Classical Approach. In Practical Raman Spectroscopy: An Introduction; 2013; pp 1-7.

(18) Schmitt, M.; Popp, J. Raman spectroscopy at the beginning of the twenty-first century. $J$. Raman Spectrosc. 2006, 37 (1-3), 20-28.

(19) Schweitzer-Stenner, R. Structure and dynamics of biomolecules probed by Raman spectroscopy. J. Raman Spectrosc. 2005, 36 (4), 276-278.

(20) Ru, E. C. Le; Etchegoin, P. G. Chapter 1 A quick overview of surface-enhanced Raman Spectroscopy. In Principles of Surface-Enhanced Raman Spectroscopy; 2008; pp 1-28. 
(21) Kneipp, K.; Kneipp, K.; Kneipp, H.; Kneipp, H.; Itzkan, I.; Itzkan, I.; Dasari, R. R.;

Dasari, R. R.; Feld, M. S.; Feld, M. S. Ultrasensitive chemical analysis by Raman spectroscopy. Chem. Rev. 1999, 99 (10), 2957-2976.

(22) G. Turrell and J. Corset. Raman Microscopy: Developments and Applications; San Diego, 1996.

(23) Séide, Marily Marion, M.; Mateescu, M. A.; Averill-Bates, D. A. The fungicide thiabendazole causes apoptosis in rat hepatocytes. Toxicol. Vitr. 2016, 32, 232-239.

(24) European Food Safety Authority. Reasoned opinion on the review of the existing maximum residue levels (MRLs) for thiabendazole according to Article 12 of Regulation (EC). EFSA J. 2014, 12 (7), 3750-3804.

(25) Young, M. S.; Phillips, D. J.; Iraneta, P. C.; Krol, J. Mixed-mode solid-phase extraction and cleanup procedures for the liquid chromatographic determination of thiabendazole and carbendazim in fruit juices. J. AOAC Int. 2001, 84 (2), 556-561.

(26) Ridgway, K.; Lalljie, S. P. D.; Smith, R. M. Sample preparation techniques for the determination of trace residues and contaminants in foods. J. Chromatogr. A 2007, 1153 (1-2), 36-53.

(27) Hennion, M. C. Solid-phase extraction: method development, sorbents, and coupling with liquid chromatography. J. Chromatogr. A 1999, 856, 3-54.

(28) Yan, H.; Row, K. H. Characteristic and Synthetic Approach of Molecularly Imprinted Polymer. Int. J. Mol. Sci. 2006, 7 (5), 155-178.

(29) Craig, A. P.; Franca, A. S.; Irudayaraj, J. Surface-enhanced Raman spectroscopy applied to food safety. Anпи. Rev. Food Sci. Technol. 2013, 4, 369-380. 
(30) Mikac, L.; Ivanda, M.; Gotic, M.; Mihelj, T.; Horvat, L. Synthesis and characterization of silver colloidal nanoparticles with different coatings for SERS application. J. Nanoparticle Res. 2014, 16 (12).

(31) Turiel, E.; Martín-Esteban, A. Molecularly imprinted stir bars for selective extraction of thiabendazole in citrus samples. J. Sep. Sci. 2012, 35 (21), 2962-2969.

(32) Barahona, F.; Turiel, E.; Martín-Esteban, A. Supported liquid membrane-protected molecularly imprinted fiber for solid-phase microextraction of thiabendazole. Anal. Chim. Acta 2011, 694 (1-2), 83-89.

(33) Díaz-Álvarez, M.; Turiel, E. Improved molecularly imprinted polymer grafted to porous polyethylene frits for the solid-phase extraction of thiabendazole from citrus sample extracts. Mol. Imprinting 2015, 2 (1), 1-7.

(34) Turiel, E.; Tadeo, J. L.; Cormack, P. a G.; Martin-Esteban, A. HPLC imprinted-stationary phase prepared by precipitation polymerization for the determination of thiabendazole in fruit. Analyst 2005, 130 (12), 1601-1607.

(35) Xiongfeng, H.; Qun, X.; Rohrer, J. Determination of Carbendazim in Orange Juice. 2013, $1-5$.

(36) Turiel, E.; Tadeo, J. L.; Cormack, P. a G.; Martin-Esteban, A. HPLC imprinted-stationary phase prepared by precipitation polymerisation for the determination of thiabendazole in fruit. Analyst 2005, 130 (12), 1601-1607.

(37) Pardeshi, S.; Dhodapkar, R.; Kumar, A. Molecularly imprinted microspheres and nanoparticles prepared using precipitation polymerisation method for selective extraction of gallic acid from Emblica officinalis. Food Chem. 2014, 146, 385-393. 
(38) Song, X.; Wang, J.; Zhu, J. Effect of porogenic solvent on selective performance of molecularly imprinted polymer for quercetin. Mater. Res. 2009, 12 (3), 299-304.

(39) Qian, K.; Fang, G.; He, J.; Pan, M.; Wang, S. Preparation and application of a molecularly imprinted polymer for the determination of trace metolcarb in food matrices by high performance liquid chromatography. J. Sep. Sci. 2010, 33 (14), 2079-2085.

(40) Dong, X.; Ji, X.; Wu, H.; Zhao, L.; Li, J.; Yang, W. Shape control of silver nanoparticles by stepwise citrate reduction. J. Phys. Chem. C 2009, 113 (16), 6573-6576.

(41) Muller, C.; David, L.; Chis, V.; Pinzaru, S. C. Detection of thiabendazole applied on citrus fruits and bananas using surface enhanced Raman scattering. Food Chem. 2014, 145, 814820.

(42) Kudelski, a; Bukowska, J.; Janik-Czachor, M.; Grochala, W.; Szummer, a; Dolata, M. Characterization of the copper surface optimized for use as a substrate for surfaceenhanced Raman scattering. Vib. Spectrosc. 1998, 16 (1), 21-29.

(43) Vandenabeele, P. 4.7 Noise in Raman spectroscopy. In Practical Ranman spectroscopy: An Introduction; 2013; pp 96-99. 


\section{Appendix}

This chapter includes all the pre-experiment data that assists to produce the final results.

In pre-experiments, we want to find the basic conditions for making standard solution and for storing the template (thiabendazole) solution, also do solvent selection test to find the best solvent for adsorption test. In Figure A. $1 \mathrm{c}$, it is obviously that there is almost no difference between the UV-absorbance spectrum for $7.5 \mathrm{ppm}$ thiabendazole solutions under different temperature. So the stock solution for thiabendazole can be stored under room temperature. Section 3.3.2 includes the results of solvent selection, and find that $20 \%$ acetonitrile will be the best choice.
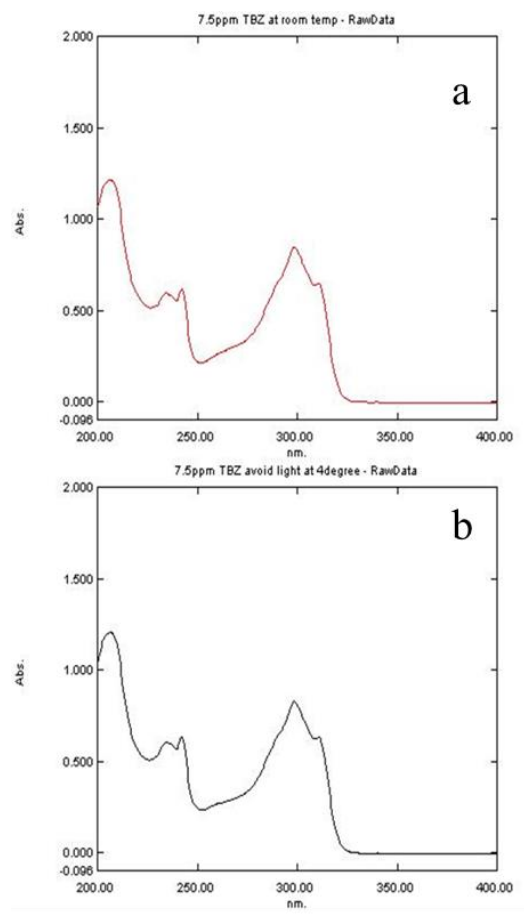
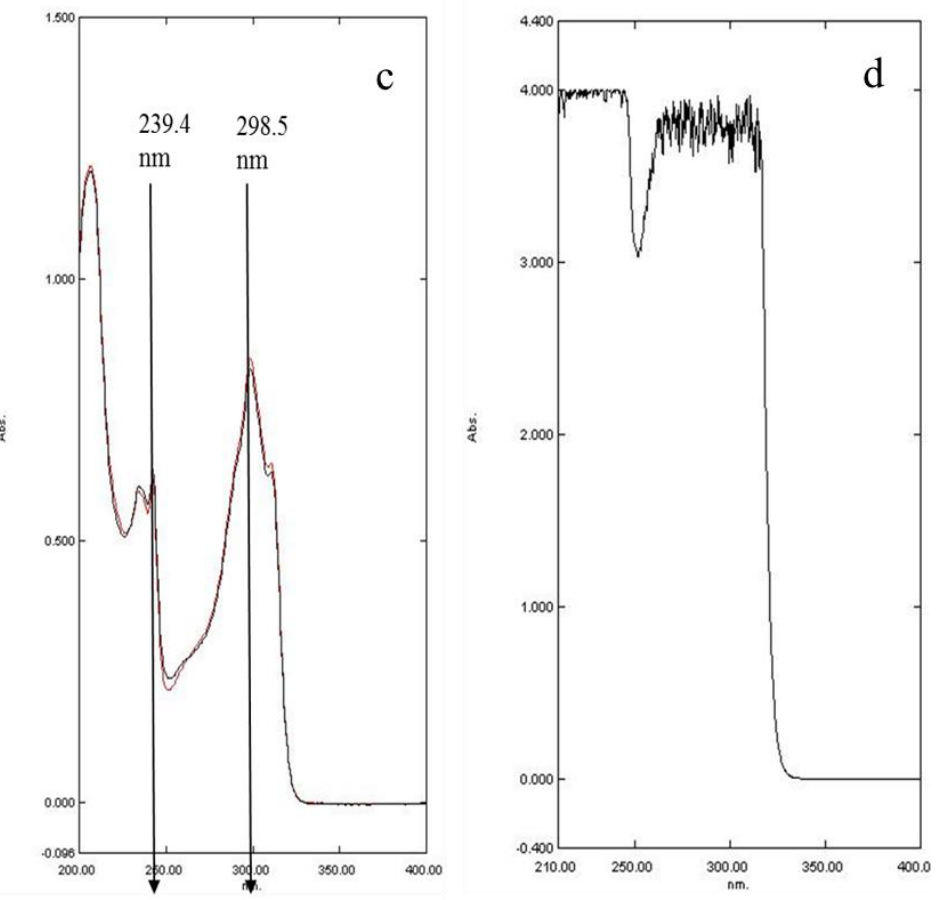

Figure A. 1 Representative UV-absorbance spectrum of thiabendazole solutions with different conditions. a) $7.5 \mathrm{ppm}$ TBZ solution store under room temperature for 24 hours. c) $7.5 \mathrm{ppm}$ TBZ solution store at $4^{\circ} \mathrm{C}$ for 24 hours. c) Compare absorbance spectrum between two conditions. d) $100 \mathrm{ppm}$ TBZ solution. 
To make a good standard curve, I tried to measure UV absorption of template solutions with different concentrations. Figure A. 1 d shows that it is overload for the UV spectrometer for 100 ppm solution. And, as we know, the best range for UV absorption is from 0.2 to 0.8 . In Figure A. 2 , with several times dilution, the selected concentrations range of thiabendazole standard solutions is from $4 \mathrm{ppm}$ to $10 \mathrm{ppm}$, where the UV-absorbance range is between 0.1 and 1 .

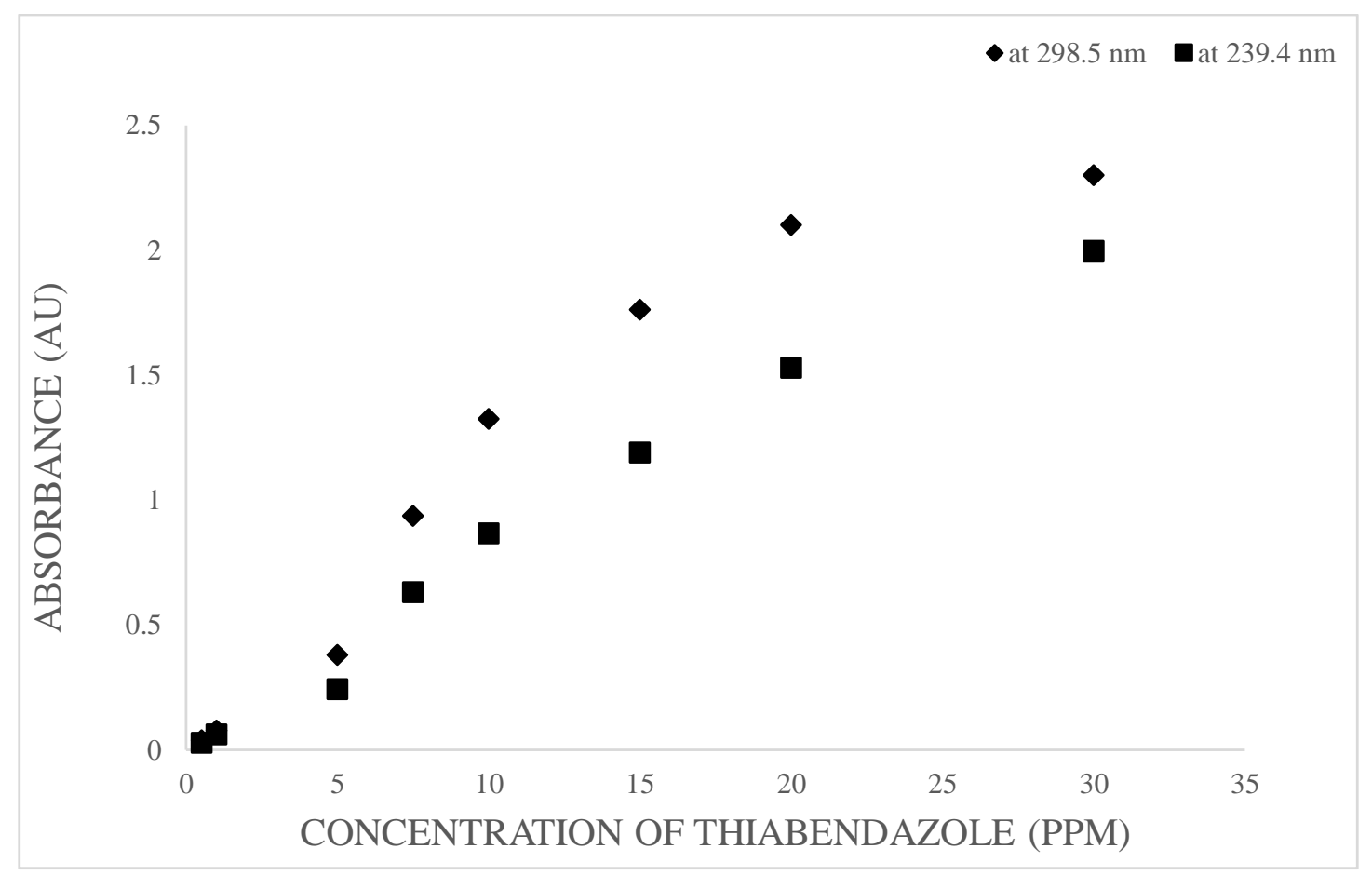

Figure A. 2 The UV absorbance of thiabendazole solution with different concentration. 
Table A. 1 Chemicals used in the MIPs synthesis process.

\begin{tabular}{llcccc}
\hline Composition & Chemicals & $\begin{array}{c}\text { Molar mass } \\
(\mathbf{m m o l})\end{array}$ & $\begin{array}{c}\text { Molar } \\
\text { ratio }\end{array}$ & $\begin{array}{c}\text { Weight } \\
(\mathbf{g})\end{array}$ & $\begin{array}{c}\text { Volume } \\
(\mathbf{m l})\end{array}$ \\
\hline Template & Thiabendazole (TBZ) & 0.17 & 1 & 0.0342 & - \\
\hline Functional & Methacrylic acid (MAA) & 0.68 & 4 & 0.0585 & 0.0577 \\
monomer & & & & & \\
\hline Cross-linker & Divinylbenzene (DVB) & 3.40 & 20 & 0.4426 & 0.4760 \\
\hline & & & & & \\
\hline Initiator & 2,2 '- & 0.25 & - & 0.4110 & - \\
& azobis(isobutyronitrile) & & & & \\
& (AIBN) & & & & \\
\hline Solvent/ & Acetonitrile : Toluene & - & - & & 12.50 \\
porogen & (75:25 v/v) & & & & \\
\hline
\end{tabular}

In Table A. 1, the name and amount of materials used in polymerization have been listed out. In page 28, the synthesis process of MIPs was illustrated in details. Figure A. 3 shows the proposed mechanism for NIPs formation. During the initiation, AIBN will be decomposed to given nitrogen gas and two free radicals under heating. In propagation, free radical initiators react with methacrylic acid or the cross-linker DVB to propagate the chain further. Combination of two active chain ends or combination of an active chain end with an initiator radical can terminate this reaction. 
$\mathrm{a}$

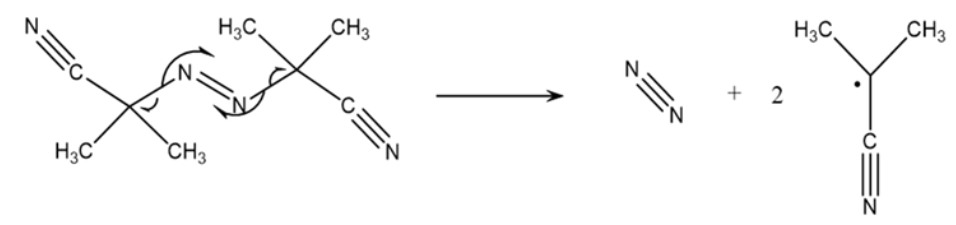

$\mathrm{b}$<smiles>COC(=O)C(C)=CCCCC(C)(C)C#N</smiles><smiles>C=Cc1ccc(C=Cc2cc(C=Cc3ccc(C=C)c(CC(C)(C)N(C)C(C)(C)C)c3)ccc2C=C)cc1</smiles>

Figure A. 3 Proposed mechanism for NIPs synthesis process. a) Initiation step: AIBN decomposed under heat and generate free radicals. b) Propagation step: Free radicals react with Methacrylic acid or the cross-linker DVB to propagate the chain further. 